\title{
BLACK'S MODEL OF INTEREST RATES AS OPTIONS, EIGENFUNCTION EXPANSIONS AND JAPANESE INTEREST RATES
}

\author{
ViATCHESLAV GOROVOI AND VADIM LINETSKY \\ Department of Industrial Engineering and Management Sciences, \\ McCormick School of Engineering and Applied Sciences, \\ Northwestern University
}

\begin{abstract}
Black's (1995) model of interest rates as options assumes that there is a shadow instantaneous interest rate that can become negative, while the nominal instantaneous interest rate is a positive part of the shadow rate due to the option to convert to currency. As a result of this currency option, all term rates are strictly positive. A similar model was independently discussed by Rogers (1995). When the shadow rate is modeled as a diffusion, we interpret the zero-coupon bond as a Laplace transform of the area functional of the underlying shadow rate diffusion (evaluated at the unit value of the transform parameter). Using the method of eigenfunction expansions, we derive analytical solutions for zero-coupon bonds and bond options under the Vasicek and shifted CIR processes for the shadow rate. This class of models can be used to model low interest rate regimes. As an illustration, we calibrate the model with the Vasicek shadow rate to the Japanese Government Bond data and show that the model provides an excellent fit to the Japanese term structure. The current implied value of the instantaneous shadow rate in Japan is negative.
\end{abstract}

KeY Words: interest rate models, Vasicek model, CIR model, bonds, bond options, eigenfunction expansion, area functional

\section{INTRODUCTION}

So long as investors can hold currency, nominal interest rates must remain nonnegative to preclude arbitrage. In practice, when interest rate derivatives are priced, Gaussian interest rate models starting with that of Vasicek (1977) are commonly employed that allow negative rates. It has often been claimed that there is no need to worry if the probability of negative rates is small. While that is true in many cases, Rogers (1996) has shown that some derivatives' prices are extremely sensitive to the possibility of negative rates. For such derivatives, the prices that the Gaussian models predict can be absurd.

An alternative class of models employs diffusion processes for the nominal instantaneous interest rate (short rate) with the property that zero is an unattainable boundary. The dynamics of the short rate are restricted to the interval $(0, \infty)$ and the short rate can never reach zero. The Brennan and Schwartz (1979) model and the popular in practice Black and Karasinski (1991) model are prominent examples. These models have a

This research was supported by the U.S. National Science Foundation under grant DMI-0200429.

Manuscript received March 2002; final revision received October 2002.

Address correspondence to Vadim Linetsky, Department of Industrial Engineering and Management Sciences, McCormick School of Engineering and Applied Sciences, Northwestern University, 2145 Sheridan Road, Evanston, IL 60208; e-mail: linetsky@iems.nwu.edu. 
"lognormal" instantaneous short-rate volatility and a mean-reverting drift. A common feature of such models is that the volatility falls off rapidly as the rate approaches zero, thus "switching off" the diffusion term and allowing the mean-reverting drift to pull the process away from zero, making zero unattainable.

The square-root Cox, Ingersoll, and Ross (1985; hereafter CIR) model is a borderline case. When the mean-reverting drift is large enough relative to the volatility, the rate cannot reach zero. Otherwise, the rate can reach zero, and one must decide on the boundary condition at zero. More precisely, if the Feller (1951) condition is satisfied, $2 \kappa \theta \geq \sigma^{2}$, zero is an unattainable entrance boundary for the process (see Section 5). Otherwise, it is an attainable regular boundary, and a boundary condition must be specified. In both cases, the short-rate volatility falls off as the square root as the short rate falls toward zero.

Such volatility structures that vanish as the short rate tends to zero contradict the accumulated empirical evidence. There have been several periods in economic history when short-term rates have fallen to zero. In the United States, this happened in the 1930s. More recently, since the mid-1990s short-term interest rates in Japan have stayed below $1 \%$, at times reaching zero. However, even though the short rates have stayed near zero, volatility has remained quite high throughout the period (Goldstein and Keirstead 1997). Obviously, both the Gaussian models that allow the rates to become negative and the lognormal or square-root models with zero an unattainable boundary and vanishing volatility are inadequate in the low interest rate regime.

Existing multifactor term structure models do not provide a solution to this problem either. To be specific, take the popular affine family of models (Dai and Singleton 2000; Duffie and Kan 1996) in which the instantaneous short rate is an affine function of an $N$-dimensional state vector of factors assumed to follow an $N$-dimensional affine diffusion process. To guarantee the nonnegativity of the short rate, all of the factors should be restricted to take nonnegative values. According to the classification of Dai and Singleton, only the subfamily $A_{N}(N)$ of correlated CIR models meets this restriction (the subfamilies $A_{m}(N)$ with $m<N$ have $N-m$ factors allowed to take negative values). The subfamily $A_{N}(N)$ contains multifactor generalizations of the CIR model. All of the factors as well as the short rate stay strictly positive, and the volatilities of all factors vanish as the factors fall toward zero.

Thus, a qualitatively different class of interest rate models is needed to model low interest rate regimes. One would like an interest rate model in which the short rate stays nonnegative (although it could become zero) and at the same time has nonvanishing volatility at low rates. Black (1995) put forward the following model of interest rates as options. Black argued that the short rate cannot become negative because currency is an option; when an instrument has a negative rate, we can choose currency instead. Therefore, we can treat the short rate itself as an option: we can choose an underlying process that can take negative values and simply replace all the negative values with zeros (take a positive part). We still have a one-factor process: either the short rate (when the underlying process is positive or zero) or what the short rate would be without the currency option (when the underlying process is negative). Black calls this process the shadow short rate. In such a model, the shadow short rate can become negative, the nominal short rate is a positive part of the shadow rate, and all term rates are strictly positive. A similar model was independently discussed by Rogers (1995).

In this paper we present analytical solutions for zero-coupon bonds and bond options in this model. When the shadow rate is modeled as a scalar diffusion, we interpret the zero-coupon bond as a Laplace transform of the area functional of the underlying shadow rate diffusion (evaluated at the unit value of the transform parameter). Using the method 
of eigenfunction expansions (Davydov and Linetsky 2003; Linetsky 2002a, 2002b), we derive analytical solutions for zero-coupon bonds and bond options under the Vasicek and shifted CIR processes for the shadow rate. As the shadow rate approaches zero, the term rate volatilities do not vanish. This class of models can be used to model low interest rate regimes.

Both Black (1995) and Rogers $(1995,1996)$ also discussed the model where the short rate is a diffusion on $[0, \infty)$ with a reflecting boundary condition at zero. Black (1995) criticized such models on economic grounds, favoring the model of interest rates as options. Models with reflecting boundary conditions at zero have been further studied in an interesting paper by Goldstein and Keirstead (1997) who obtained solutions for zero-coupon bond prices in terms of eigenfunction expansions for some specifications of the process. These authors argued that Black's model of interest rates as options has the disadvantage that it is analytically intractable, whereas the model with a reflecting boundary at zero admits analytical solutions for zero-coupon bonds and European-style interest rate derivatives. In this paper we show that, in fact, Black's model of interest rates as options is as fully analytically tractable as the reflecting boundary model, and we obtain analytical solutions both for zero-coupon bonds and bond options under several specifications for the shadow rate process. Thus, there is no need to restrict oneself to the model with the reflecting boundary at zero in order to retain analytical tractability.

The remainder of the paper is organized as follows. In Section 2 we describe the model. The shadow rate is modeled as a diffusion that is allowed to become negative. The short rate is a positive part of this process. To facilitate analytical solutions for bond options, we also impose an additional assumption that the spectrum of the infinitesimal generator of the pricing semigroup is purely discrete. We interpret the zero-coupon bond in this model as a Laplace transform of the area functional (evaluated at the unit value of the transform parameter). In Section 3 we outline the eigenfunction expansion approach to the problem of pricing bonds and bond options in this model. In Section 4 we specify the Vasicek process for the shadow rate and find explicit solutions for zero-coupon bonds and bond options in terms of eigenfunction expansions. In Section 5 we obtain analytical solutions for the shifted CIR process for the shadow rate. Computational results are presented in Section 6. For longer times to maturity the eigenfunction expansions converge fast, with several terms producing values accurate to five or six decimals. In Section 7 we calibrate the model with the Vasicek shadow rate process to the Japanese Government Bond (JGB) data and show that the model provides an excellent fit to the Japanese term structure. Section 8 concludes the paper. The Appendix contains the proofs.

\section{THE MODEL}

\subsection{The Shadow Short-Rate Process}

In this paper we take an equivalent martingale measure $Q$ as given. We assume that, under $Q$, the shadow interest rate follows a one-dimensional, regular time-homogeneous diffusion process $\left\{X_{t}, t \geq 0\right\}$ taking values in some interval $I$ with the endpoints $l$ and $r,-\infty \leq l \leq 0<r \leq \infty$, and with the infinitesimal generator

$$
(\mathcal{G} f)(x)=\frac{1}{2} \sigma^{2}(x) f^{\prime \prime}(x)+\mu(x) f^{\prime}(x), \quad x \in(l, r),
$$

acting on functions on $I$ with appropriate regularity and boundary conditions. We assume that the diffusion and drift parameters satisfy the following assumptions: $\sigma, \sigma^{\prime}, \sigma^{\prime \prime}, \mu$, 
and $\mu^{\prime}$ are continuous ${ }^{1}$ in the open interval $(l, r)$ and $\sigma(x)>0$ in $(l, r)$. Furthermore, we assume that the speed density of the diffusion satisfies the following conditions ${ }^{2}$ :

$$
\int_{l}^{\epsilon} \mathfrak{m}(x) d x<\infty, \quad \int_{\epsilon}^{r} \mathfrak{m}(x) d x<\infty, \quad \epsilon \in(l, r) .
$$

The scale and speed densities $\mathfrak{s}$ and $\mathfrak{m}$ of the diffusion $X$ are defined by ${ }^{3}$

$$
\begin{gathered}
\mathfrak{s}(x):=\exp \left\{-\int^{x} \frac{2 \mu(y)}{\sigma^{2}(y)} d y\right\} \\
\mathfrak{m}(x):=\frac{2}{\sigma^{2}(x) \mathfrak{s}(x)} .
\end{gathered}
$$

Conditions (2.2) guarantee that the diffusion has a stationary density (see Karlin and Taylor 1981, pp. 241-242):

$$
\pi(x)=\frac{\mathfrak{m}(x)}{\int_{l}^{r} \mathfrak{m}(y) d y} .
$$

Under conditions (2.2) the left $(l)$ and right $(r)$ endpoints can be regular, entrance, or nonattracting natural boundaries. ${ }^{4}$ To be specific, consider the left endpoint $l$. Under condition (2.2) at $l$, it is a regular boundary if the following integral is finite:

$$
\int_{l}^{\epsilon} \mathfrak{s}(x) d x<\infty, \quad \epsilon \in(l, r) .
$$

Otherwise, it is either entrance or nonattracting natural. Define a scale function $\left(x_{0} \in(l, r)\right.$ can be selected arbitrarily; the scale function is defined up to an additive constant):

$$
S(x):=\int_{x_{0}}^{x} \mathfrak{s}(y) d y, \quad x_{0} \in(l, r) .
$$

If condition (2.2) is satisfied and the integral in equation (2.6) is infinite, then $l$ is entrance if the following integral is finite:

$$
\int_{l}^{\epsilon} S(x) \mathfrak{m}(x) d x<\infty, \quad \epsilon \in(l, r) .
$$

It is nonattracting natural if the integral in equation (2.8) is infinite. Similar classification results hold for the right endpoint $r$. We assume that infinite boundaries are unattainable. In this paper we always make regular boundaries instantaneously reflecting. Regular reflecting boundaries are included in the state space $I$. Entrance and natural boundaries are not included in $I$ (e.g., if $l$ is regular reflecting and $r$ is natural, then $I=[l, r)$ ).

\footnotetext{
${ }^{1}$ We will need these smoothness assumptions in order to transform to the Liouville normal form in Section 3.1.

2 These conditions are standard in the interest rate literature (e.g., Conley, Hansen, Luttmer, and Scheinkman 1997).

${ }^{3}$ Our definition of the speed density coincides with those of Karatzas and Shreve (1991, p. 343) and Borodin and Salminen (1996, p. 17) and differs from Karlin and Taylor (1981, p. 194) who do not include 2 in the definition.

${ }^{4}$ For Feller's boundary classification for one-dimensional diffusions see Karlin and Taylor (1981, Chap. 15) and Borodin and Salminen (1996, Chap. 2).
} 


\subsection{The Nominal Short-Rate Process}

Following Black (1995) and Rogers (1995), we assume that the nominal instantaneous interest rate (the short rate) $\left\{r_{t}, t \geq 0\right\}$ is a positive part of the shadow rate:

$$
r_{t}=X_{t}^{+}, \quad t \geq 0 .
$$

The left boundary $-\infty \leq l \leq 0$ is either $-\infty$ or a fixed nonpositive real. In the limiting case $l=0$ the shadow rate coincides with the nominal rate and is always nonnegative. The right endpoint $0<r \leq+\infty$ is either a positive real or $+\infty$.

\subsection{Zero-Coupon Bonds and Bond Options}

A zero-coupon bond pays one dollar at a prespecified time $T>0$. Then the price (present value) of the zero-coupon bond at some time $0 \leq t \leq T$ is given by the risk-neutral expectation

$$
P(x, t, T)=E_{t, x}\left[e^{-\int_{t}^{T} r_{u} d u}\right]=E_{t, x}\left[e^{-\int_{t}^{T} X_{u}^{+} d u}\right],
$$

where $E_{t, x}[\cdot] \equiv E\left[\cdot \mid X_{t}=x\right]$ (for $t=0$ we drop the 0 in $E_{0, x}$ and write $E_{x}$ ). Since the shadow rate process is time homogeneous, we have

$$
P(x, t, T)=P(x, 0, \tau)=E_{x}\left[e^{-\int_{0}^{\tau} X_{u}^{+} d u}\right]=: P(x, \tau), \quad \tau:=T-t .
$$

The corresponding yield-to-maturity $R(x, \tau)$ is defined as usual:

$$
R(x, \tau):=-\frac{1}{\tau} \ln P(x, \tau), \quad \tau>0 .
$$

Call and put options with the expiration date $t$ and strike price $K$ written on the zero-coupon bond with maturity $T>t$ deliver the payoffs at time $t$ :

$$
\left(P\left(X_{t}, t, T\right)-K\right)^{+}, \quad\left(K-P\left(X_{t}, t, T\right)\right)^{+},
$$

respectively. Caplets and floorlets in interest rate caps and floors can be represented as options on zero-coupon bonds (Hull 2000 and James and Weber 2000). Since we are working with one-factor models, options on coupon-bearing bonds and swaptions can be expressed as portfolios of options on zero-coupon bonds using the approach of Jamshidian (1989). We thus limit ourselves to options on zero-coupon bonds.

\subsection{Model Interpretation and Hedging}

Since the short rate is always nonnegative, $r_{t} \geq 0$, the zero-coupon bond prices $P(x, \tau)$ given by equation (2.11) are always strictly less than one for any $\tau>0$. Moreover, for each fixed $x$ the zero-coupon bond pricing function $\{P(x, \tau), \tau \geq 0\}$ is strictly decreasing on $[0, \infty)$ with $P(x, 0)=1$ and all term rates (or yields) $R(x, \tau)$ as well as all forward rates $f(x, \tau), f(x, \tau)=-\frac{\partial}{\partial \tau} \ln P(x, \tau)$ (Heath, Jarrow, and Morton 1992) are strictly positive for all $\tau>0$. This ensures that the model is free from the arbitrage opportunities present in models that admit negative rates, as discussed in the Introduction.

We stress that the underlying fundamental economic factor (state variable) in this model is the shadow rate $x$, not the short rate $r$ : the model is Markovian in the shadow rate and not in the short rate. For hedging purposes, one computes sensitivities of interest rate derivatives to the shadow rate $x$. Strictly speaking, there is no sense in talking about sensitivities with respect to the short rate $r$ since the short rate is not a Markovian 
state variable in this model (value functions of interest rate derivatives in this model are functions of $x$ ). When the shadow rate is strictly positive, the short rate is equal to the shadow rate, and one can interpret sensitivities of interest rate derivatives to the shadow rate computed at positive values of the latter as sensitivities to the short rate. However, when the shadow rate is nonpositive, it makes sense to talk about sensitivities of interest rate derivatives to the shadow rate, while sensitivities to the short rate are undefined. If one selects a zero-coupon bond with some maturity $T>0$ as a hedging instrument in this model (since this is a single-factor model, one hedging instrument is sufficient), at time $t<T$ any other interest rate derivative $V$ can be hedged with

$$
h=\frac{V_{x}(t, x)}{P_{x}(x, t, T)}
$$

units of the zero-coupon bond, where $V_{x}$ and $P_{x}$ are the partials with respect to the shadow rate of the interest rate derivative value function and the zero-coupon bond, respectively.

We also note that, since for any fixed $\tau>0$ the term rate (yield) $R_{\tau}:=R(x, \tau)$ is a positive and strictly increasing function of the shadow rate $x$, one can uniquely invert this one-to-one relationship to express the shadow rate $x$ as a function of the term rate $R_{\tau}$ for any fixed $\tau>0$. Then one can compute sensitivities of interest rate derivatives to the term rate $R_{\tau}$. In contrast, the relationship between the shadow rate and the short rate is not one-to-one (the short rate is not a sufficient statistic of the state in this model).

\subsection{Area Functionals of Scalar Diffusions}

We note that the zero-coupon bond price (2.11) has the form of the Laplace transform (evaluated at the unit value of the transform parameter) of an area functional of the shadow rate diffusion:

$$
A_{t}:=\int_{0}^{t} X_{u}^{+} d u, \quad t \geq 0 .
$$

The area functional measures the area below the positive part of a sample path of the diffusion process up to time $t$. Perman and Wellner (1996) studied area functionals of Brownian motion. In our application we are interested in area functionals of onedimensional diffusions with stationary densities. In particular, in this paper we will compute the zero-coupon bond price (Laplace transform evaluated at the unit value of the transform parameter)

$$
P(x, t)=E_{x}\left[e^{-A_{t}}\right]
$$

and the put option price

$$
E_{x}\left[e^{-A_{t}}\left(K-P\left(X_{t}, T-t\right)\right)^{+}\right] .
$$

The call option price is obtained from the put-call parity for bond options:

$$
\begin{aligned}
E_{x}\left[e^{-A_{t}}\left(P\left(X_{t}, T-t\right)-K\right)^{+}\right]= & E_{x}\left[e^{-A_{t}}\left(K-P\left(X_{t}, T-t\right)\right)^{+}\right] \\
& +P(x, T)-K P(x, t) .
\end{aligned}
$$

We thus limit ourselves to put options on zero-coupon bonds. 
REMARK 2.1. The area functional joins several other additive functionals important in mathematical finance. Among them are averages and exponential Brownian functionals (e.g., Dufresne 2000, 2001; Geman and Yor 1993; Linetsky 2001; Yor 2001) local times (e.g., Carr and Jarrow 1990), and occupation times (e.g., Chesney et al. 1997; Davydov and Linetsky 2002; Linetsky 1998, 1999). For Brownian motion with drift, the area functional has already appeared in finance in connection with the valuation of executive stock options in Carr and Linetsky (2000).

\section{THE EIGENFUNCTION EXPANSION APPROACH}

To compute prices of zero-coupon bonds and interest rate derivatives we employ the spectral expansion approach described in detail in Linetsky (2002b). In this section we extensively use the results in Linetsky (2002b) and refer the reader to that paper for details, proofs, and further references.

\subsection{The General Framework}

Let $\mathcal{H}:=L^{2}(I, \mathfrak{m})$ be the Hilbert space of real-valued functions square-integrable on $I$ with the speed density $\mathfrak{m}(x)$ and with the inner product

$$
(f, g)=\int_{l}^{r} f(x) g(x) \mathfrak{m}(x) d x .
$$

Let $\left\{\mathcal{P}_{t}, t \geq 0\right\}$ be the self-adjoint contraction semigroup in $\mathcal{H}$ associated with the shadow rate process $\left\{X_{t}, t \geq 0\right\}$,

$$
\left(\mathcal{P}_{t} f\right)(x):=E_{x}\left[f\left(X_{t}\right)\right], \quad f \in \mathcal{H}, t \geq 0, x \in I,
$$

and let $\mathcal{G}$ be its infinitesimal generator,

$$
(\mathcal{G} f)(x)=\frac{1}{2} \sigma^{2}(x) f^{\prime \prime}(x)+\mu(x) f^{\prime}(x), \quad x \in(l, r) .
$$

If an endpoint $e \in\{l, r\}$ is regular instantaneously reflecting, then $(\mathcal{G} f)(e):=$ $\lim _{x \rightarrow e}(\mathcal{G} f)(x)$. The generator can be expressed in terms of the scale and speed densities:

$$
(\mathcal{G} f)(x)=\frac{1}{\mathfrak{m}(x)}\left(\frac{f^{\prime}(x)}{\mathfrak{s}(x)}\right)^{\prime} .
$$

Its domain $D(\mathcal{G})$ is $D(\mathcal{G}):=\left\{f \in \mathcal{H}: f, f^{\prime} \in A C_{\text {loc }}(I), \mathcal{G} f \in \mathcal{H}\right.$, boundary conditions at $l$ and $r$, where $A C_{l o c}(I)$ is the space of functions absolutely continuous over each compact subinterval of $I$, and the appropriate boundary conditions at $l$ and $r$ are:

$$
\lim _{x \downarrow l} \frac{f^{\prime}(x)}{\mathfrak{s}(x)}=0, \quad \lim _{x \uparrow r} \frac{f^{\prime}(x)}{\mathfrak{s}(x)}=0 .
$$

The infinitesimal generator $\mathcal{G}$ is self-adjoint and nonpositive in $\mathcal{H}$. We are interested in its spectrum and the associated spectral representation for the transition density $p(t ; x, y)$,

$$
\left(\mathcal{P}_{t} f\right)(x)=\int_{l}^{r} f(y) p(t ; x, y) d y .
$$

Furthermore, let $\left\{\hat{\mathcal{P}}_{t}, t \geq 0\right\}$ be the self-adjoint contraction semigroup (pricing semigroup) of discounted conditional expectation operators (pricing or present value operators) in $\mathcal{H}$,

$$
\left(\hat{\mathcal{P}}_{t} f\right)(x):=E_{x}\left[e^{-A_{t}} f\left(X_{t}\right)\right], \quad f \in \mathcal{H}, t \geq 0, x \in I
$$


and let $\hat{\mathcal{G}}$ be its infinitesimal generator,

$$
\hat{\mathcal{G}}=\mathcal{G}-x^{+} .
$$

We can view $\hat{\mathcal{G}}$ as the infinitesimal generator of a diffusion process $\hat{X}$ with the killing rate $x^{+}$. Feller's boundary classification can be extended to diffusions with killing (see Borodin and Salminen 1996, pp. 14-15). The nature of the endpoint $l$ is not changed since the killing rate $x^{+}$vanishes below zero. If $r<+\infty$, the killing rate is continuous on the closed interval $[0, r]$. Hence, adding the killing does not change the nature of the finite right endpoint and $D(\hat{\mathcal{G}})=D(\mathcal{G})$ (see Karlin and Taylor 1981, Thm. 12.3, p. 314). If $r=+\infty$ is a natural boundary for the diffusion without killing, it remains natural for the diffusion with killing. If $r=+\infty$ is an entrance boundary for the diffusion without killing, it can either remain entrance or become natural for the diffusion with killing. If $r=+\infty$, then the domain $D(\hat{\mathcal{G}})$ is $D(\hat{\mathcal{G}}):=\left\{f \in \mathcal{H}: f, f^{\prime} \in A C_{l o c}(I), \hat{\mathcal{G}} f \in \mathcal{H}\right.$, boundary conditions at $l$ and $r$. The boundary conditions at $l$ and $r$ remain the same as for the diffusion without killing (equation (3.5)).

The operator $\hat{\mathcal{G}}$ is self-adjoint and nonpositive in $\mathcal{H}$. We are interested in its spectrum and the associated spectral representation for the state-price density $q(t ; x, y)$,

$$
\left(\hat{\mathcal{P}}_{t} f\right)(x)=\int_{l}^{r} f(y) q(t ; x, y) d y .
$$

REMARK 3.1. Note that if we do not take the positive part and allow the nominal rate to become negative, the resulting pricing semigroup is, in general, no longer a contraction semigroup, and its infinitesimal generator is, in general, no longer nonpositive. This may result in absurd economic consequences, as we will see later in the Vasicek example.

Consider the two associated Sturm-Liouville (SL) ordinary differential equations:

$$
-(\mathcal{G} u)(x)=\lambda u(x), \quad x \in(l, r)
$$

and

$$
-(\hat{\mathcal{G}} u)(x) \equiv-(\mathcal{G} u)(x)+x^{+} u(x)=\lambda u(x), \quad x \in(l, r)
$$

with the boundary conditions (3.5). We are interested in spectral expansions in $\mathcal{H}$ associated with these Sturm-Liouville problems.

More generally, consider a SL equation ${ }^{5}$ with $\lambda \in \mathbb{C}$ and $x \in(l, r)$ :

$$
-\frac{1}{\mathfrak{m}(x)}\left(\frac{u^{\prime}(x)}{\mathfrak{s}(x)}\right)^{\prime}+r(x) u(x)=\lambda u(x),
$$

with some $r(x)$ continuous and nonnegative on $(l, r)$. The oscillatory/nonoscillatory classification based on Sturm's theory of oscillations of solutions is of fundamental importance in determining the qualitative nature of the spectrum of the SL problem. For a

\footnotetext{
${ }^{5}$ See Dunford and Schwartz (1963), Glazman (1965), Levitan and Sargsjan (1975), Weidmann (1987), and a recent survey by Fulton, Pruess, and Xie (1996) for the Sturm-Liouville theory. Applications of spectral theory to diffusion processes were initiated by McKean (1956) (see also Ito and McKean 1974, Sect. 4.11, and Langer and Schenk 1990). Applications to derivatives pricing can be found in Beaglehole (1991), Davydov and Linetsky (2003), Goldstein and Keirstead (1997), Lewis (1994, 1998, 2000), Linetsky (2001, 2002a, 2002b, 2003), and Lipton (2001). Applications to estimation of diffusion processes in econometrics can be found in Hansen, Scheinkman, and Touzi (1998). In this paper we follow Linetsky (2002b).
} 
given real $\lambda$, equation (3.12) is oscillatory at an endpoint $e$ if and only if every solution has infinitely many zeros clustering at $e$. Otherwise it is called nonoscillatory at $e$. This classification is mutually exclusive for a fixed $\lambda$, but can vary with $\lambda$. For equation (3.12), there are two distinct possibilities at each endpoint. Let $e \in\{l, r\}$ be an endpoint of equation (3.12). Then $e$ belongs to one and only one of the following two cases: (i) (NONOSC) Equation (3.12) is nonoscillatory at $e$ for all real $\lambda$. Correspondingly, $e$ is called nonoscillatory or NONOSC for brevity. (ii) (O-NO) There exists a real number $\Lambda \geq 0$ such that equation (3.12) is oscillatory at $e$ for all $\lambda>\Lambda$ and nonoscillatory at $e$ for all $\lambda<\Lambda$. Correspondingly, $e$ is called $O-N O$ with cutoff $\Lambda$. Equation (3.12) can be either oscillatory or nonoscillatory at $e$ for $\lambda=\Lambda>0$. It is always nonoscillatory for $\lambda=0$.

If a boundary for diffusion with killing is regular, exit, or entrance in Feller's classification, it is NONOSC for the Sturm-Liouville equation associated with its infinitesimal generator. Feller's natural boundaries can be either NONOSC or O-NO with nonnegative cutoff $\Lambda \geq 0$. If both endpoints are NONOSC, then the spectrum of the SL problem is simple, nonnegative, and purely discrete. If one or both endpoints are O-NO, then there is some nonempty continuous spectrum and the associated eigenfunction expansion in $\mathcal{H}$ includes an integral with respect to the continuous part of the spectral measure. As we will see in Section 3.2, this complicates the pricing of bond options. Since in this paper we are interested in pricing both bonds and bond options, we limit ourselves to such shadow rate diffusions that the SL problem (3.11) associated with the infinitesimal generator $\hat{\mathcal{G}}$ with the diffusion (killing) rate $x^{+}$has a purely discrete spectrum. Both the Vasicek and shifted CIR processes of Sections 4 and 5 fall in this category. Thus, we make an additional assumption that both boundaries are NONOSC for the SL equation (3.11).

Generally, to determine when an endpoint of equation (3.12) is NONOSC or O-NO with cutoff $\Lambda$, it is convenient to transform the SL equation to the Liouville normal form (LNF). The following smoothness assumption is sufficient to be able to perform the Liouville transform: $\sigma^{\prime}(x), \sigma^{\prime \prime}(x)$, and $\mu^{\prime}(x)$ exist and are continuous on $(l, r)$. Fix some $x_{0} \in(l, r)$ and transform the independent and dependent variables as follows:

$$
\begin{gathered}
y=\sqrt{2} \int_{x_{0}}^{x} \frac{d z}{\sigma(z)}, \\
v(y)=\frac{2^{\frac{1}{4}} u(x(y))}{\sqrt{\sigma(x(y)) \mathfrak{s}(x(y))}},
\end{gathered}
$$

where $x=x(y)$ is the inverse of the transformation $y=y(x)$. Then the function $v(y)$ satisfies the SL equation in the Liouville normal form (the coefficient in front of the second derivative is constant and equal to (negative) one and the first-derivative term is absent; the SL equation in the Liouville normal form is called the one-dimensional Schrödinger equation with potential function $Q(y))$ :

$$
-v^{\prime \prime}+Q(y) v=\lambda v, \quad y \in(y(l), y(r)),
$$

where the potential function $Q(y)$ is given by

$$
Q(y)=\mathcal{V}(x(y))
$$

where

$$
\mathcal{V}(x):=\frac{1}{8}\left(\sigma^{\prime}(x)\right)^{2}-\frac{1}{4} \sigma(x) \sigma^{\prime \prime}(x)+\frac{\mu^{2}(x)}{2 \sigma^{2}(x)}+\frac{1}{2} \mu^{\prime}(x)-\frac{\mu(x) \sigma^{\prime}(x)}{\sigma(x)}+r(x) .
$$


The oscillatory/nonoscillatory classification remains invariant under the Liouville transform - that is, equation (3.12) is nonoscillatory at $e \in\{l, r\}$ for a particular $\lambda$ if and only if equation (3.15) is nonoscillatory at $y(e)$ for that $\lambda$. The oscillatory/nonoscillatory classification of equation (3.12) depends on the behavior of the potential function near the endpoints. We cite the following classification result (see Linetsky (2002b for the proof and relevant references).

Proposition 3.1 (NONOSC/O-NO Classification). Let $e \in\{l, r\}$ be an endpoint of the $S L$ equation (3.12) and let $\mathcal{V}(x)$ be the function defined by (3.17). Assume that the limit $\lim _{x \rightarrow e} \mathcal{V}(x)$ exists (it is allowed to be infinite).

(i) If e is transformed into a finite endpoint by the Liouville transform-that is, $y(e)=$ $\sqrt{2} \int_{x_{0}}^{e} \frac{d z}{\sigma(z)}$ is finite - then e is NONOSC.

(ii) Suppose e is transformed into $-\infty$ or $+\infty$ by the Liouville transform. If

$$
\lim _{x \rightarrow e} \mathcal{V}(x)=+\infty
$$

then e is NONOSC. If for some finite $\Lambda$

$$
\lim _{x \rightarrow e} \mathcal{V}(x)=\Lambda,
$$

then e is $O$-NO with cutoff $\Lambda$.

Since in this paper we wish to limit ourselves to shadow rate diffusions for which the pricing semigroup has a purely discrete spectrum, we assume that if $e \in\{l, r\}$ is transformed into an infinite endpoint by the Liouville transform, then the diffusion and drift parameters $\sigma(x)$ and $\mu(x)$ are such that $\lim _{x \rightarrow e} \mathcal{V}(x)=+\infty$ recall that $\left(r(x)=x^{+}\right.$in this case). Then the spectral representation of the state-price density is ${ }^{6}$ :

$$
q(t ; x, y)=\mathfrak{m}(y) \sum_{n=0}^{\infty} e^{-\lambda_{n} t} \varphi_{n}(x), \varphi_{n}(y), \quad x, y \in I, t>0,
$$

where $\left\{\lambda_{n}\right\}_{n=0}^{\infty}, 0<\lambda_{0}<\lambda_{1}<\cdots, \lim _{n \uparrow \infty} \lambda_{n}=\infty$, are the eigenvalues and $\left\{\varphi_{n}(x)\right\}_{n=0}^{\infty}$ are the corresponding eigenfunctions of the Sturm-Liouville problem (3.11) normalized so that $\left\|\varphi_{n}\right\|^{2}=1$. The eigenfunctions form a complete, orthonormal basis in the Hilbert space $\mathcal{H}$. The $\varphi_{n}(x)$ are also eigenfunctions of the pricing operator:

$$
\left(\hat{\mathcal{P}}_{t} \varphi_{n}\right)(x) \equiv E_{x}\left[e^{-A_{t}} \varphi_{n}\left(X_{t}\right)\right]=e^{-\lambda_{n} t} \varphi_{n}(x), \quad x \in I, t \geq 0 .
$$

That is, the present value at time zero of the payoff $\varphi_{n}\left(X_{t}\right)$ paid at time $t \geq 0$ is just $e^{-\lambda_{n} t} \varphi_{n}(x)$, where $x$ is the initial value of the state variable (shadow rate) at time zero. The corresponding contingent claims with eigenpayoffs $\varphi_{n}$ are called eigensecurities in the terminology of Davydov and Linetsky (2003).

Now consider some payoff $f \in \mathcal{H}$ to be paid at time $t \geq 0$. We have the following eigenfunction expansion:

$$
f(x)=\sum_{n=0}^{\infty} c_{n} \varphi_{n}(x), \quad c_{n}=\left(f, \varphi_{n}\right),
$$

\footnotetext{
${ }^{6}$ In Linetsky (2002b) we label the eigenvalues $\lambda_{n}$ starting from $n=1$. Here, for future convenience in Sections 4 and 5 , we label the eigenvalues starting from $n=0$.
} 
the Parseval equality holds, $\|f\|^{2}=\sum_{n=0}^{\infty} c_{n}^{2}$, and convergence is in the norm of the Hilbert space. For the present value of any payoff $f \in \mathcal{H}$ we have the associated eigenfunction expansion:

$$
\left(\hat{\mathcal{P}}_{t} f\right)(x) \equiv E_{x}\left[e^{-A_{t}} f\left(X_{t}\right)\right]=\sum_{n=0}^{\infty} c_{n} e^{-\lambda_{n} t} \varphi_{n}(x), \quad x \in I, t \geq 0 .
$$

REMARK 3.2. Note that it is possible that the spectrum of $\mathcal{G}$ contains some nonempty continuous spectrum, while the spectrum of $\hat{\mathcal{G}}$ is purely discrete. Brennan and Schwartz's (1979) model provides such an example. The negative of the infinitesimal generator of the diffusion $d X_{t}=\kappa\left(\theta-X_{t}\right) d t+\sigma X_{t} d B_{t}$ on $(0, \infty)$ with positive parameters $\kappa, \theta$, and $\sigma$ has a continuous spectrum above $\Lambda=\frac{1}{2 \sigma^{2}}\left(\kappa-\sigma^{2} / 2\right)^{2}$ plus a finite number of nonnegative discrete eigenvalues below $\Lambda$. However, the negative of the infinitesimal generator of the pricing semigroup, $-\hat{\mathcal{G}}=-\mathcal{G}+x$, that includes discounting (killing) has a purely discrete positive spectrum.

\subsection{Pricing Zero-Coupon Bonds and Bond Options}

To price zero-coupon bonds, consider constant payoffs. Due to conditions (2.2), constants are in the Hilbert space $\mathcal{H}$, therefore the above analysis applies and we have an eigenfunction expansion for the zero-coupon bond price:

$$
\begin{gathered}
P(x, t)=E_{x}\left[e^{-A_{t}}\right]=\sum_{n=0}^{\infty} c_{n} e^{-\lambda_{n} t} \varphi_{n}(x), \\
c_{n}=\int_{l}^{r} \varphi_{n}(x) \mathfrak{m}(x) d x .
\end{gathered}
$$

Due to the factors $e^{-\lambda_{n} t}$, the longer the time to maturity, the faster the eigenfunction expansion converges.

REMARK 3.3. From (3.24) we have the following asymptotics for large times to maturity:

$$
\lim _{t \uparrow \infty} R(x, t)=\lim _{t \uparrow \infty}\left(-\frac{1}{t} \ln P(x, t)\right)=\lambda_{0} \geq 0 .
$$

As time to maturity increses, the yield curve flattens out and approaches the principal eigenvalue $\lambda_{0}>0$ (see Lewis 1994, 1998 for general discussions along these lines, as well as some interesting examples). In Black's model of interest rates as options, the principal eigenvalue is guaranteed to be strictly positive. However, in models that allow negative nominal rates the principal eigenvalue can, in general, be negative. This can lead to absurd economic consequences. As time to maturity increases, the zero-coupon bond price (discount factor) blows up to infinity as the yield curve flattens out and approaches a negative asymptotic yield. We will see this phenomenon in the Vasicek model in Section 4.

We now turn to the pricing of bond options.

Proposition 3.2. The price of a put option expiring at time $t>0$ written on a zerocoupon bond maturing at $T>t$ has the eigenfunction expansion

$$
E_{x}\left[e^{-A_{t}}\left(K-P\left(X_{t}, T-t\right)\right)^{+}\right]=\sum_{n=0}^{\infty} p_{n}(\tau) e^{-\lambda_{n} t} \varphi_{n}(x)
$$


with coefficients

$$
p_{n}(\tau)=K A_{n}-\sum_{m=0}^{\infty} c_{m} B_{n, m} e^{-\lambda_{m} \tau}, \quad \tau:=T-t,
$$

where $c_{m}$ are the coefficients of the zero-coupon bond expansion (3.25),

$$
A_{n}:=\int_{x^{*}}^{\infty} \varphi_{n}(x) \mathfrak{m}(x) d x, \quad B_{n, m}:=\int_{x^{*}}^{\infty} \varphi_{n}(x) \varphi_{m}(x) \mathfrak{m}(x) d x,
$$

and $x^{*}$ is the unique root of the equation (the zero-coupon bond price $P(x, \tau)$ has the eigenfunction expansion (3.24)):

$$
K-P\left(x^{*}, T-t\right)=0 .
$$

Proof. See the Appendix.

The longer the time to option expiration $t$ and the longer the time between the option expiration and the maturity of the underlying bond $\tau=T-t$, the faster the eigenfunction expansion (3.27-3.28) converges (due to the presence of the factors $e^{-\lambda_{n} t}$ in (3.27) and $e^{-\lambda_{m} \tau}$ in (3.28)).

REMARK 3.4. Note that if there is some nonempty continuous spectrum, the eigenfunction expansion (3.24) for the bond price includes an integral in place of the sum. Then the eigenfunction expansion (3.27-3.28) for the bond option includes a double integral in place of the sums in (3.27) and (3.28). This double integral has to be evaluated numerically. To avoid these computational complications, in this paper we restrict ourselves to shadow rate processes that lead to purely discrete spectra for the pricing semigroup.

\subsection{Determining Eigenvalues and Eigenfunctions}

The problem of pricing contingent claims is now reduced to the problem of finding eigenvalues $\lambda_{n}$ and eigenfunctions $\varphi_{n}$ of the Sturm-Liouville problem (3.11). We assume that equation (3.11) is nonoscillatory at $l$ and $r$ for all real $\lambda$ to guarantee purely discrete spectrum.

Proposition 3.3. Suppose $-\infty \leq l<0<r \leq+\infty$. Let $\psi_{\lambda}(x), x \in(l, 0], \lambda \in \mathbb{C}$, be the unique (up to a multiple independent of $x$ ) solution of the $O D E(3.11)$ on the interval $(l, 0]$,

$$
-\frac{1}{2} \sigma^{2}(x) u^{\prime \prime}(x)-\mu(x) u^{\prime}(x)=\lambda u(x), \quad x \in(l, 0]
$$

such that

$$
\int_{l}^{0}\left|\psi_{\lambda}(x)\right|^{2} \mathfrak{m}(x) d x<\infty
$$

and

$$
\lim _{x \downarrow l} \frac{\psi_{\lambda}^{\prime}(x)}{\mathfrak{s}(x)}=0
$$

for each $\lambda \in \mathbb{C}$, and $\psi_{\lambda}(x)$ and $\psi_{\lambda}^{\prime}(x) \equiv \frac{d \psi_{\lambda}(x)}{d x}$ are continuous in $x$ and $\lambda$ in $(l, 0] \times \mathbb{C}$ and entire in $\lambda$ for each fixed $x \in(l, 0]$. 
Let $\phi_{\lambda}(x), x \in[0, r), \lambda \in \mathbb{C}$, be the unique (up to a multiple independent of $x$ ) solution of the $O D E$ (3.11) on the interval $[0, r)$,

$$
-\frac{1}{2} \sigma^{2}(x) u^{\prime \prime}(x)-\mu(x) u^{\prime}(x)+x u(x)=\lambda u(x), \quad x \in(0, r]
$$

such that

$$
\int_{0}^{r}\left|\phi_{\lambda}(x)\right|^{2} \mathfrak{m}(x) d x<\infty
$$

and

$$
\lim _{x \uparrow r} \frac{\phi_{\lambda}^{\prime}(x)}{\mathfrak{s}(x)}=0
$$

for each $\lambda \in \mathbb{C}$, and $\phi_{\lambda}(x)$ and $\phi_{\lambda}^{\prime}(x)=\frac{d \phi_{\lambda}(x)}{d x}$ are continuous in $x$ and $\lambda$ in $[0, r) \times \mathbb{C}$ and entire in $\lambda$ for each fixed $x \in[0, r)$.

Define a function of the complex variable $\lambda$ :

$$
w(\lambda):=\frac{1}{\mathfrak{s}(0)}\left(\psi_{\lambda}(0) \phi_{\lambda}^{\prime}(0)-\phi_{\lambda}(0) \psi_{\lambda}^{\prime}(0)\right) .
$$

It is an entire function of $\lambda$ with all of its zeros simple and located on the positive real half-line. The eigenvalues $0<\lambda_{0}<\lambda_{1}<\cdots$ of the problem (3.11) can be identified with (positive, simple) roots of the equation

$$
w(\lambda)=0,
$$

and the corresponding continuous eigenfunctions with continuous first derivatives and normalized so that $\left\|\varphi_{n}\right\|^{2}=1$ are:

$$
\varphi_{n}(x)=\left\{\begin{array}{ll}
\sqrt{\frac{\phi_{\lambda_{n}}(0)}{\Delta_{n} \psi_{\lambda_{n}}(0)}} \psi_{\lambda_{n}}(x), & l<x \leq 0 \\
\sqrt{\frac{\phi_{\lambda_{n}}(0)}{\Delta_{n} \phi_{\lambda_{n}}(0)}} \phi_{\lambda_{n}}(x), & 0 \leq x<r
\end{array},\right.
$$

where

$$
\Delta_{n}:=\left.\frac{d w(\lambda)}{d \lambda}\right|_{\lambda=\lambda_{n}}
$$

Proof. See the Appendix.

Thus, combining the results of Sections 3.2 and 3.3, we have expressed both the bond and bond option pricing formulas in terms of the solutions $\psi_{\lambda}(x)$ and $\phi_{\lambda}(x)$ of the ODEs (3.31) and (3.34) in the intervals $(l, 0]$ and $[0, r)$, respectively.

For comparison, we now provide the corresponding results for the reflecting boundary model. This model assumes that the short rate follows a diffusion process on $[0, r)$ with the infinitesimal generator (2.1) and instantaneously reflecting boundary at zero. Zerocoupon bonds and bond options are given by the eigenfunction expansions (3.24) and (3.27-3.28) with eigenvalues and eigenfunctions described in the following proposition.

Proposition 3.4. Let $\phi_{\lambda}(x)$ be as in Proposition 3.3. Then the eigenvalues $0<\lambda_{0}<$ $\lambda_{1}<\cdots$ can be identified with (positive, simple) roots of the equation

$$
\phi_{\lambda}^{\prime}(0)=0
$$


and the corresponding continuous eigenfunctions with continuous first derivatives and normalized so that $\left\|\varphi_{n}\right\|^{2}=1$ are

$$
\varphi_{n}(x)=\frac{\phi_{\lambda_{n}}(x)}{\sqrt{\Delta_{n} \phi_{\lambda_{n}}(0)}}, \quad x \in[0, r), \quad \text { where } \Delta_{n}:=\left.\frac{d \phi_{\lambda}^{\prime}(0)}{d \lambda}\right|_{\lambda=\lambda_{n}} .
$$

Proof. See the Appendix.

REMARK 3.5. In the finance literature it is sometimes assumed that to normalize eigenfunctions one has to start with some nonnormalized eigenfunctions $\tilde{\varphi}_{n}$, compute the norms by numerical integration, $\left\|\tilde{\varphi}_{n}\right\|^{2}=\int_{l}^{r} \tilde{\varphi}_{n}^{2}(x) \mathfrak{m}(x) d x$, and then normalize the eigenfunctions $\varphi_{n}(x)=\tilde{\varphi}_{n}(x) /\left\|\tilde{\varphi}_{n}\right\|$. In fact, the eigenfunction norms can be expressed analytically and no numerical integration is necessary. The eigenfunctions in Propositions 3.3 and 3.4 are normalized. See Linetsky (2002b) for details.

\section{THE VASICEK PROCESS FOR THE SHADOW RATE}

Our first example is the Vasicek (1977) or Ornstein-Uhlenbeck process for the shadow rate:

$$
d X_{t}=\kappa\left(\theta-X_{t}\right) d t+\sigma d B_{t}, \quad X_{0}=x,
$$

where $\theta>0$ is the long-run level of the shadow rate, $\kappa>0$ is the rate of mean reversion toward the long-run level, and $\sigma>0$ is the volatility parameter. When the process is below $\theta$, the positive drift pulls it back up toward $\theta$. When the process is above $\theta$, the negative drift pulls it back down toward $\theta$. For this process $l=-\infty$ and $r=+\infty$. The scale and speed densities are

$$
\mathfrak{s}(x)=e^{\frac{\kappa(\theta-x)^{2}}{\sigma^{2}}}, \quad \mathfrak{m}(x)=\frac{2}{\sigma^{2}} e^{-\frac{\kappa(\theta-x)^{2}}{\sigma^{2}}},
$$

both $-\infty$ and $+\infty$ are nonattracting natural boundaries, and the process has a stationary distribution with the Gaussian density

$$
\pi(x)=\sqrt{\frac{\kappa}{\pi \sigma^{2}}} e^{-\frac{\kappa(\theta-x)^{2}}{\sigma^{2}}} .
$$

The Liouville transformation (3.13-3.14) with $x_{0}=\theta$ reduces the associated SturmLiouville equation (3.10) to the stationary Schrödinger equation (3.12) with the harmonic oscillator potential

$$
Q(y)=\frac{\kappa^{2}}{4} y^{2}-\frac{\kappa}{2}
$$

The potential increases to $+\infty$ as $y \rightarrow \pm \infty$ and, hence, both endpoints are NONOSC. The spectrum is purely discrete and nonnegative. The transition density $p(t ; x, y)$ has a well-known spectral representation of the form (3.20) with the eigenvalues and normalized eigenfunctions (e.g., Karlin and Taylor 1981, p. 333):

$$
\begin{gathered}
\lambda_{n}=\kappa n, \quad \varphi_{n}(x)=\mathcal{N}_{n} H_{n}(\xi), \quad n=0,1, \ldots, \quad \text { where } \xi:=\frac{\sqrt{\kappa}}{\sigma}(x-\theta), \\
\mathcal{N}_{n}^{2}=\sqrt{\frac{\kappa}{\pi}} \frac{\sigma}{2^{n+1} n !},
\end{gathered}
$$


where $H_{n}(x)$ are Hermite polynomials. Applying Mehler's formula (eqn. (22) in Erdelyi 1953, p. 194)

$$
\sum_{n=0}^{\infty} \frac{(z / 2)^{n}}{n !} H_{n}(x) H_{n}(y)=\left(1-z^{2}\right)^{-1 / 2} \exp \left\{\frac{2 x y z-\left(x^{2}+y^{2}\right) z^{2}}{1-z^{2}}\right\},
$$

the spectral representation reduces to the familiar Gaussian density

$$
p(t ; x, y)=\sqrt{\frac{\kappa}{\pi \sigma^{2}\left(1-e^{-2 \kappa t}\right)}} \exp \left\{-\frac{\kappa\left(x e^{-\kappa t}-y+\theta\left(1-e^{-\kappa t}\right)\right)^{2}}{\sigma^{2}\left(1-e^{-2 \kappa t}\right)}\right\} .
$$

Next consider the Sturm-Liouville equation with discounting,

$$
-\frac{1}{2} \sigma^{2} u^{\prime \prime}-\kappa(\theta-x) u^{\prime}+x u=\lambda u,
$$

on the whole real line. The Liouville transformation reduces it to the Schrödinger equation with potential

$$
Q(y)=\frac{\kappa^{2}}{4}\left(y+\frac{\sqrt{2} \sigma}{\kappa^{2}}\right)^{2}-\frac{\kappa}{2}+\left(\theta-\frac{\sigma^{2}}{2 \kappa^{2}}\right) .
$$

The potential increases to $+\infty$ as $y \rightarrow \pm \infty$ and, hence, both endpoints are NONOSC. The spectrum is purely discrete and bounded from below. However, it is no longer guaranteed to be nonnegative. The Vasicek state-price density $q_{V}(t ; x, y)$,

$$
E_{x}\left[e^{-\int_{0}^{t} X_{u} d u} f\left(X_{t}\right)\right]=\int_{-\infty}^{\infty} f(y) q_{V}(t ; x, y) d y
$$

has a spectral representation of the form (3.20) with the eigenvalues and normalized eigenfunctions ( $\xi$ and $\mathcal{N}_{n}$ are as in equations (4.3), (4.4))

$$
\lambda_{n}=\theta-\frac{\sigma^{2}}{2 \kappa^{2}}+\kappa n, \quad \varphi_{n}(x)=\mathcal{N}_{n} e^{-a \xi-\frac{a^{2}}{2}} H_{n}(\xi+a), \quad n=0,1, \ldots, a:=\frac{\sigma}{\kappa^{3 / 2}} .
$$

Integrating the unit payoff against this state-price density, we have the eigenfunction expansion of the form (3.24-3.25) for the zero-coupon bond price with the expansion coefficients

$$
c_{n}=\frac{2}{\sigma} \sqrt{\frac{\pi}{\kappa}} \mathcal{N}_{n} a^{n} e^{-\frac{a^{2}}{4}} .
$$

Applying Mehler's formula, the spectral representation reduces to the familiar expression for the Vasicek state-price density

$$
q_{V}(t ; x, y)=e^{-\lambda_{0} t-\frac{1}{\kappa}(x+y-2 \theta)-\frac{\sigma^{2}}{\kappa^{3}}} p\left(t ; x+\sigma^{2} / \kappa^{2}, y+\sigma^{2} / \kappa^{2}\right),
$$

where $p(t ; x, y)$ is the Gaussian transition density (4.5). Applying the formula for the generating function of Hermite polynomials (eqn. (19) in Erdelyi 1953, p. 194)

$$
\sum_{n=0}^{\infty} \frac{z^{n}}{n !} H_{n}(x)=e^{2 x z-z^{2}}
$$

the eigenfunction expansion for the Vasicek bond price collapses to the familiar Vasicek bond pricing formula

$$
P_{V}(x, \tau)=e^{-\tau R(x, \tau)}, \quad R(x, \tau)=\lambda_{0}+\left(x-\lambda_{0}\right) \frac{1-e^{-\kappa \tau}}{\kappa \tau}+\frac{\sigma^{2}}{4 \kappa^{3} \tau}\left(1-e^{-\kappa \tau}\right)^{2},
$$


where $\lambda_{0}=\theta-\frac{\sigma^{2}}{2 \kappa^{2}}$ is the principal eigenvalue. The principal eigenvalue $\lambda_{0}$ is negative when $2 \kappa^{2} \theta<\sigma^{2}$. As time to maturity increases, the yield curve flattens out and approaches the negative principal eigenvalue and the zero-coupon bond price blows up to infinity.

In contrast with the original Vasicek (1977) model, according to Black's model of interest rates as options the instantaneous nominal rate is a positive part of the shadow rate process (4.1). Thus, the instantaneous nominal rate is always nonnegative and all term rates (yields on zero-coupon bonds) are strictly positive. We now turn to the pricing of bonds and bond options in this model.

Proposition 4.1. Introduce the following notation:

$$
\begin{gathered}
z:=\frac{\sqrt{2 \kappa}}{\sigma}(\theta-x), \quad \alpha:=\sigma \sqrt{\frac{2}{\kappa^{3}}}, \quad \beta:=\frac{\sqrt{2 \kappa}}{\sigma} \theta, \\
v(\lambda):=\frac{\lambda}{\kappa}, \quad \mu(\lambda):=\frac{1}{\kappa}\left(\lambda-\theta+\frac{\sigma^{2}}{2 \kappa^{2}}\right) .
\end{gathered}
$$

The functions $\psi_{\lambda}(x)$ and $\phi_{\lambda}(x)$ of Proposition 3.3 are expressed in terms of the Weber parabolic cylinder function $D_{v}(z)$ (we follow the notation of Erdelyi 1953, pp. 116-130, and Buchholz 1969, pp. 39-49):

$$
\begin{gathered}
\psi_{\lambda}(x)=e^{\frac{z^{2}}{4}} D_{v(\lambda)}(z), \quad x \in(-\infty, 0], \\
\phi_{\lambda}(x)=e^{\frac{z^{2}}{4}} D_{\mu(\lambda)}(\alpha-z), \quad x \in[0, \infty) .
\end{gathered}
$$

Using the differential properties of the Weber function, the function $w(\lambda)$ takes the form:

$$
\begin{aligned}
\omega(\lambda)= & c v(\lambda) D_{\mu(\lambda)}(\alpha-\beta) D_{\nu(\lambda)-1}(\beta) \\
& +c D_{\nu(\lambda)}(\beta)\left[\mu(\lambda) D_{\mu(\lambda)-1}(\alpha-\beta)-\frac{\alpha}{2} D_{\mu(\lambda)}(\alpha-\beta)\right], \quad \text { where } c:=\frac{\sqrt{2 \kappa}}{\sigma} .
\end{aligned}
$$

Proof. See the Appendix.

The Weber-Hermite parabolic cylinder function $D_{v}(z)$ is expressed in terms of the Hermite function $H_{v}(z)$ (Lebedev (1972), p. 284):

$$
D_{v}(z)=2^{-\frac{v}{2}} e^{-\frac{z^{2}}{4}} H_{v}\left(\frac{z}{\sqrt{2}}\right)
$$

When $v=n$ is an integer, the Hermite function reduces to the Hermite polynomial. For any $v$, real or complex, the Hermite function is available as a built-in function in Mathematica (HermiteH[ $[v, z]$ ) and can be computed with arbitrarily high level of precision. For all computations in this paper we used Mathematica 4.0 running on a Pentium III PC. The eigenvalues are determined by finding (positive, simple) zeros of the function $w(\lambda)$. To compute the eigenfunction expansion coefficients, the single integrals in equations (3.25) and (3.29) were computed numerically using the built-in numerical integration routine in Mathematica. The Vasicek model with the reflecting boundary 
condition at zero was implemented similarly by substituting the function (4.11) in Proposition 3.4.

\section{THE SHIFTED CIR PROCESS FOR THE SHADOW RATE}

Our second example is a shifted Feller $(1951)$ and CIR diffusion on $(l, \infty)^{7}$ :

$$
d X_{t}=\kappa\left(\theta-X_{t}\right) d t+\sigma \sqrt{X_{t}-l} d B_{t},
$$

where $\theta>0$ is the "long-run level", $\kappa>0$ is the rate of mean reversion, and $\sigma>0$ is the volatility parameter. When $l=0$ we have the standard CIR process. Here we are interested in the shifted CIR process with $l<0$. We assume that the parameters satisfy Feller's condition $2 \kappa(\theta-l) \geq \sigma^{2}$. The scale and speed densities are:

$$
\mathfrak{s}(x)=(x-l)^{-\beta} e^{\frac{2 \kappa(x-l)}{\sigma^{2}}}, \quad \mathfrak{m}(x)=\frac{2}{\sigma^{2}}(x-l)^{\beta-1} e^{-\frac{2 \kappa(x-l)}{\sigma^{2}}}, \quad \beta:=\frac{2 \kappa(\theta-l)}{\sigma^{2}} .
$$

Under Feller's condition $\beta \geq 1$, conditions (2.2) and (2.6) are satisfied, $l$ is entrance, $+\infty$ is nonattracting natural, and the process has a stationary distribution with the shifted Gamma density

$$
\pi(x)=\frac{2 \kappa}{\sigma^{2} \Gamma(\beta)}\left(\frac{2 \kappa(x-l)}{\sigma^{2}}\right)^{\beta-1} e^{-\frac{2 \kappa(x-l)}{\sigma^{2}}} .
$$

The Liouville transformation (3.13-3.14) with $x_{0}=l$ reduces the associated SturmLiouville equation (3.10) into the Schrödinger equation $(3.15)$ on $(0, \infty)$ with the so-called radial harmonic oscillator potential:

$$
Q(y)=\frac{[3 / 4+\beta(\beta-2)]}{y^{2}}-\frac{\kappa \beta}{2}+\frac{\kappa^{2} y^{2}}{16} .
$$

The potential increases to $+\infty$ as $x \uparrow+\infty$ and, hence, $+\infty$ is NONOSC. Since both endpoints are NONOSC, the spectrum is purely discrete, and the transition probability density $p(t ; x, y)$ of the CIR process has a well-known spectral representation of the form (3.20) with the eigenvalues and eigenfunctions (e.g., Wong 1964 and Davydov and Linetsky 2003):

$$
\lambda_{n}=\kappa n, \quad \varphi_{n}(x)=\mathcal{N}_{n} L_{n}^{(\beta-1)}(\xi), \quad n=0,1, \ldots, \quad \text { where } \xi:=\frac{2 \kappa(x-l)}{\sigma^{2}},
$$

$$
\mathcal{N}_{n}^{2}=\left(\frac{2 \kappa}{\sigma^{2}}\right)^{\beta-1} \frac{n ! \kappa}{\Gamma(\beta+n)}
$$

where $L_{n}^{(\alpha)}(z)$ are the generalized Laguerre polynomials. Applying the Hille-Hardy formula (eqn. (20) in Erdelyi 1953, p. 189); $I_{\alpha}(x)$ is the modified Bessel function of order $\alpha$ :

$$
\sum_{n=0}^{\infty} \frac{z^{n} n !}{\Gamma(n+\alpha+1)} L_{n}^{(\alpha)}(x) L_{n}^{(\alpha)}(y)=(1-z)^{-1} \exp \left\{-z \frac{x+y}{1-z}\right\}(x y z)^{-\frac{\alpha}{2}} I_{\alpha}\left(\frac{2 \sqrt{x y z}}{1-z}\right),
$$

\footnotetext{
${ }^{7}$ See also Goeing-Jaeschke and Yor (2003), Pitman and Yor (1982), Shiga and Watanabe (1973) for more details on these processes.
} 
the spectral representation reduces to the familiar transition density for the shifted Feller (1951) diffusion ( $\xi$ is defined in equation (5.4) and $\left.\eta:=\frac{2 \kappa(y-l)}{\sigma^{2}}\right)$ :

$$
p(t ; x, y)=\frac{2 \kappa}{\sigma^{2}\left(1-e^{-\kappa t}\right)}\left(\frac{\eta}{\xi e^{-\kappa t}}\right)^{\frac{\beta-1}{2}} \exp \left\{-\frac{\eta+\xi e^{-\kappa t}}{1-e^{-\kappa t}}\right\} I_{\beta-1}\left(\frac{2 \sqrt{\xi \eta e^{-\kappa t}}}{1-e^{-\kappa t}}\right) .
$$

Now consider the Sturm-Liouville equation for the shifted CIR process with discounting

$$
-\frac{1}{2} \sigma^{2}(x-l) u^{\prime \prime}-\kappa(\theta-x) u^{\prime}+x u=\lambda u
$$

on $(l, \infty)$ (allowing for negative rates). The Liouville transform reduces it to the Schrödinger equation with the radial harmonic oscillator potential

$$
Q(y)+l+\frac{\sigma^{2} y^{2}}{8}
$$

where $Q(y)$ is the potential (5.3) without discounting. The spectrum is again purely discrete. The shifted CIR state-price density $q_{\mathrm{CIR}}(t ; x, y)$ has a spectral representation of the form (3.20) with the eigenvalues and eigenfunctions (Davydov and Linetsky 2003; $\xi$ and $\mathcal{N}_{n}$ are defined in equations $\left.(5.4-5.5)\right)$ :

$$
\begin{gathered}
\lambda_{n}=\gamma n+\frac{\beta}{2}(\gamma-\kappa), \quad \text { where } \gamma:=\sqrt{\kappa^{2}+2 \sigma^{2}}, . \\
\varphi_{n}(x)=\mathcal{N}_{n}\left(\frac{\gamma}{\kappa}\right)^{\frac{\beta}{2}} e^{\frac{(\kappa-\gamma) x}{\sigma^{2}}} L_{n}^{(\beta-1)}\left(\frac{\gamma \xi}{\kappa}\right) .
\end{gathered}
$$

Integrating the unit payoff against this state-price density, we obtain the eigenfunction expansion of the form (3.24) for the zero-coupon bond price with the expansion coefficients (Davydov and Linetsky 2003; $\mathcal{N}_{n}$ is defined by equation (5.8)):

$$
c_{n}=\mathcal{N}_{n} \frac{2 \Gamma(\beta+n)}{\sigma^{2} n !}\left(\frac{\gamma}{\kappa}\right)^{\frac{\beta}{2}}\left(\frac{\sigma^{2}}{\gamma+\kappa}\right)^{\beta}\left(\frac{\kappa-\gamma}{\kappa+\gamma}\right)^{n} .
$$

Applying the Hille-Hardy formula, the spectral representation reduces to the closed-form expression for the shifted CIR state-price density similar to the transition density (5.6):

$$
\begin{aligned}
q_{\mathrm{CIR}}(t ; x, y)= & e^{-\lambda_{0} t+\frac{(\kappa-\gamma)}{\sigma^{2}}(x-y+2 l)} \\
& \times \frac{2 \gamma}{\sigma^{2}\left(1-e^{-\gamma t}\right)}\left(\frac{\eta}{\xi e^{-\gamma t}}\right)^{\frac{\beta-1}{2}} \exp \left\{-\frac{\gamma\left(\eta+\xi e^{-\gamma t}\right)}{\kappa\left(1-e^{-\gamma t}\right)}\right\} I_{\beta-1}\left(\frac{2 \gamma \sqrt{\xi \eta e^{-\gamma t}}}{\kappa\left(1-e^{-\gamma t}\right)}\right) .
\end{aligned}
$$

Applying the well-known formula for the generating function of Laguerre polynomials (eqn. (17) in Erdelyi 1953, p. 189)

$$
\sum_{n=0}^{\infty} z^{n} L_{n}^{(\alpha)}(x)=(1-z)^{-\alpha-1} \exp \left\{\frac{x z}{z-1}\right\}
$$

the eigenfunction expansion for the shifted CIR bond price collapses to the shifted CIR bond pricing formula ${ }^{8}$

$$
P(x, T)=A(T) e^{-l T-B(T)(x-l)},
$$

\footnotetext{
${ }^{8}$ We note that essentially the same expression was independently obtained by Pitman and Yor (1982) and by Wong (1964) in entirely different contexts.
} 


$$
B(T):=\frac{2\left(e^{\gamma T}-1\right)}{(\gamma+\kappa)\left(e^{\gamma T}-1\right)+2 \gamma}, \quad A(T):=\left(\frac{2 \gamma e^{(\kappa+\gamma) T / 2}}{(\gamma+\kappa)\left(e^{\gamma T}-1\right)+2 \gamma}\right)^{\beta} .
$$

In this shifted CIR model, in contrast to the standard CIR, the process is allowed to become negative. However, the principal eigenvalue $\lambda_{0}$ is always positive. Hence, the asymptotic yield is postive as well.

We now turn to Black's model of interest rates as options where the nominal rate is a positive part of the shifted CIR shadow rate.

Proposition 5.1. Let $-\infty<l<0$. Introduce the following additional notation:

$$
\kappa_{1}(\lambda):=\frac{\lambda}{\kappa}+\frac{\beta}{2}, \quad \kappa_{2}(\lambda):=\frac{\lambda-l}{\gamma}+\frac{\beta \kappa}{2 \gamma}, \quad m:=\frac{\beta-1}{2}, \quad \alpha:=-\frac{2 \kappa l}{\sigma^{2}} .
$$

The functions $\psi_{\lambda}(x)$ and $\phi_{\lambda}(x)$ are expressed in terms of the Whittaker functions $M_{k, m}(z)$ and $W_{k, m}(z)$ (Abramowitz and Stegun 1972, p. 505; Buchholz 1969, pp. 9-20; Slater 1960, pp. 9-10):

$$
\begin{array}{cc}
\psi_{\lambda}(x)=(x-l)^{-\frac{\beta}{2}} e^{\frac{\kappa(x-l)}{\sigma^{2}}} M_{k_{1}(\lambda), m}(\xi), & x \in(l, 0], \\
\phi_{\lambda}(x)=(x-l)^{-\frac{\beta}{2}} e^{\frac{\kappa(x-l)}{\sigma^{2}}} W_{k_{2}(\lambda), m}\left(\frac{\gamma \xi}{\kappa}\right), & x \in[0, \infty),
\end{array}
$$

where $\xi$ is defined in Eq. (73) and $\gamma$ is defined in (75). Using the differential properties of the Whittaker functions, the function $w(\lambda)$ takes the form

$$
\begin{aligned}
\omega(\lambda)= & \frac{1}{|l|} M_{k_{1}(\lambda), m}(\alpha)\left[W_{k_{2}(\lambda)+1, m}\left(\frac{\gamma \alpha}{\kappa}\right)-\left(\frac{\gamma \alpha}{2 \kappa}-k_{2}(\lambda)\right) W_{k_{2}(\lambda), m}\left(\frac{\gamma \alpha}{\kappa}\right)\right] \\
& +\frac{1}{|l|} W_{k_{2}(\lambda), m}\left(\frac{\gamma \alpha}{\kappa}\right)\left[\left(\frac{1}{2}+m+k_{1}(\lambda)\right) M_{k_{1}(\lambda)+1, m}(\alpha)-\left(k_{1}(\lambda)-\frac{\alpha}{2}\right) M_{k_{1}(\lambda), m}(\alpha)\right] .
\end{aligned}
$$

Proof. See the Appendix.

The Whittaker functions $M$ and $W$ are related to the Kummer confluent hypergeometric functions (both are available as built-in functions in Mathematica)

$$
\begin{gathered}
M_{k, m}(z)=z^{\frac{1}{2}+m} e^{-\frac{z}{2}} M(1 / 2+m-k, 1+2 m, z), \\
W_{k, m}(z)=z^{\frac{1}{2}+m} e^{-\frac{z}{2}} U(1 / 2+m-k, 1+2 m, z) .
\end{gathered}
$$

To compute the coefficients in the eigenfunction expansions for bonds and bond options, the single integrals in equations (3.25) and (3.29) are computed numerically using the built-in numerical integration routine in Mathematica.

\section{COMPUTATIONAL RESULTS}

Table 6.1 presents computational results for zero-coupon bonds in Black's models with Vasicek and shifted CIR shadow rates. For longer times to maturity several terms are enough to attain accuracy of five significant digits. As time to maturity decreases, more terms in the eigenfunction expansion have to be added to attain the same accuracy. This convergence pattern is the opposite of typical convergence patterns of numerical PDE solvers and Monte Carlo simulation. 
TABLE 6.1

Convergence of Eigenfunction Expansions for Zero-Coupon Bonds in Black's Model of Interest Rates as Options

\begin{tabular}{|c|c|c|c|c|}
\hline \multirow[b]{2}{*}{$x$} & \multicolumn{4}{|c|}{ Maturity (years) } \\
\hline & 1 & 5 & 10 & 30 \\
\hline \multicolumn{5}{|c|}{ Black's model with Vasicek shadow rate } \\
\hline $1 \%$ & $0.98829(38)$ & $0.92449(11)$ & $0.84104(5)$ & $0.58363(3)$ \\
\hline $0 \%$ & $0.99463(25)$ & $0.94622(14)$ & $0.87124(7)$ & $0.61258(3)$ \\
\hline \multicolumn{5}{|c|}{ Original Vasicek } \\
\hline $1 \%$ & 0.99011 & 0.95679 & 0.93577 & 1.01986 \\
\hline $0 \%$ & 0.99958 & 0.99518 & 0.99684 & 1.12152 \\
\hline \multicolumn{5}{|c|}{ Black's model with shifted CIR shadow rate } \\
\hline $1 \%$ & $0.98848(14)$ & $0.92763(7)$ & $0.85165(4)$ & $0.62735(3)$ \\
\hline $0 \%$ & $0.99464(25)$ & $0.94756(6)$ & $0.87812(5)$ & $0.64978(3)$ \\
\hline \multicolumn{5}{|c|}{ Original shifted CIR } \\
\hline $1 \%$ & 0.99012 & 0.95770 & 0.93869 & 0.98046 \\
\hline $0 \%$ & 0.99958 & 0.99515 & 0.99529 & 1.05768 \\
\hline
\end{tabular}

Note: Two underlying processes are considered: Vasicek with parameters $\theta=$ $0.01(1 \%), k=0.1$, and $\sigma_{\text {Vasicek }}=0.02$ and shifted CIR with parameters $\theta=0.01, \kappa=$ 0.1 , and $l=-0.05(-5 \%)$. To facilitate comparison, the volatility parameter $\sigma_{\mathrm{CIR}}$ for the shifted CIR model is selected so that at the initial shadow rate level $x$ the absolute volatility is the same as the volatility of the Vasicek process, $\sigma_{\mathrm{CIR}} \sqrt{x-l}=\sigma_{\text {Vasicek }}=$ 0.02 . Two starting shadow rate values are considered: $x=1 \%$ and $0 \%$. Numbers in parentheses next to the bond prices give the number of terms in the eigenfunction expansion required to attain accuracy of five significant digits. The original Vasicek and shifted CIR bond prices are computed using the standard closed-form Vasicek and shifted CIR bond pricing formulas.

Table 6.2 presents computational results for bond options. The shadow rate is assumed to follow the Vasicek process with parameters $\theta=0.01(1 \%), \kappa=0.1$, and $\sigma_{\text {Vasicek }}=0.02$. The put option considered in this example expires in two years $(t=2)$ and is written on a four-year zero-coupon bond $(T=4)$; therefore, at the time the option expires the underlying bond will have two years remaining to maturity $(\tau=T-t=2)$. The initial shadow rate is $x=0.01$. The option is at-the-money at inception (i.e., the strike is equal to the forward bond price $K=P(x, 0,4) / P(x, 0,2)=0.9666928)$. The corresponding value of $x^{*}$ is $1.52853 \%$. The series in equation (3.27) is truncated after $N$ terms. The series in equation (3.28) is truncated after $M$ terms. The table gives the corresponding put option values. The corresponding original Vasicek put option value is 0.01093 .

Figure 6.1 plots four sample yield curves in Black's model with the Vasicek shadow rate process and initial shadow rates $x=1 \%, 0 \%,-1 \%$, and $-5 \%$. The model parameters are $\theta=0.01, \kappa=0.1$, and $\sigma=0.02$. The yield curve with $x=1 \%$ starts at $1 \%$ (the short rate is equal to the shadow rate) and gradually increases toward the asymptotic yield equal to the principal eigenvalue $\lambda_{0}=0.017423$ (see equation (3.26)). This yield curve has a typical upward-sloping shape. The curve with the initial shadow rate of $0 \%$ starts at zero (the short rate is equal to the shadow rate), quickly increases toward $1 \%$, then the rate of increase slows and the curve gradually tends toward the asymptotic yield $\lambda_{0}$. The curve with $x=-1 \%$ has a negative initial shadow rate. It starts at zero then gradually increases 
TABLE 6.2

Convergence of Eigenfunction Expansions for Bond Options in Black's Model of Interest Rates as Options

\begin{tabular}{ccccc}
\hline & $M=1$ & 5 & 10 & 15 \\
\hline$N=1$ & 0.06878 & 0.01607 & 0.01604 & 0.01604 \\
5 & 0.06149 & 0.01260 & 0.01256 & 0.01255 \\
10 & 0.05784 & 0.01162 & 0.01163 & 0.01161 \\
20 & 0.05737 & 0.01152 & 0.01153 & 0.01152 \\
25 & 0.05735 & 0.01151 & 0.01152 & $\mathbf{0 . 0 1 1 5 1}$ \\
\hline
\end{tabular}

Note: The shadow rate is assumed to follow the Vasicek process with parameters $\theta=$ $0.01(1 \%), \kappa=0.1$, and $\sigma=0.02$. The put option expires in two years $(t=2)$ and is written on a four-year zero-coupon bond $(T=4)$; therefore, at the time the option expires the underlying bond will have two years remaining to maturity ( $\tau=T-t=$ 2). The initial shadow rate is $x=0.01$. The option is at-the-money at inception (i.e., the strike is equal to the forward price $K=P(x, 0,4) / P(x, 0,2)=0.9666928)$. The corresponding value of $x^{*}$ is $1.52853 \%$. The series in equation (3.27) is truncated after $N$ terms. The series in equation (3.28) is truncated after $M$ terms. The table gives the corresponding put option values. The corresponding original Vasicek put option value is 0.01093 .

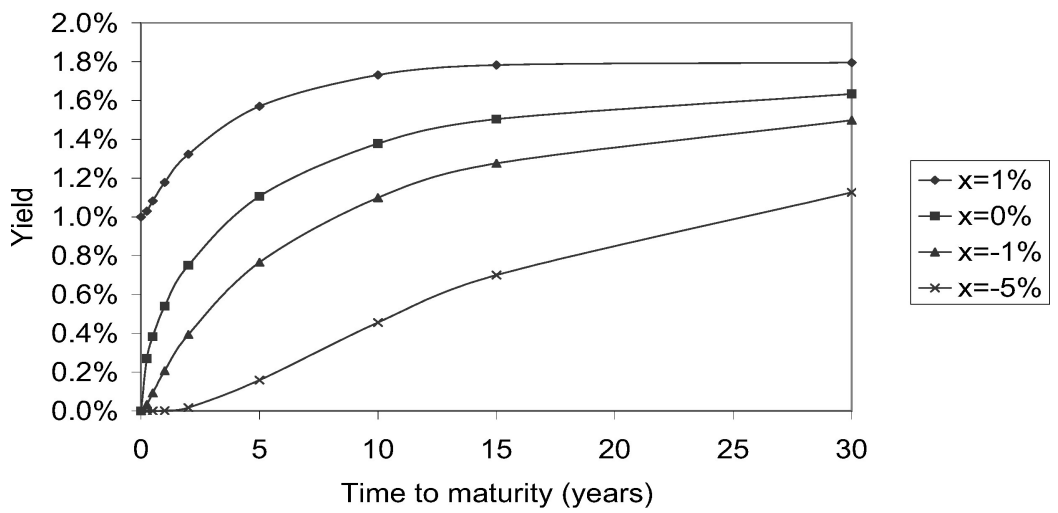

FIGURE 6.1. Yield curves in Black's model with the Vasicek shadow rate. Parameters: $\theta=0.01, \kappa=0.1, \sigma=0.02$, and initial shadow rates $x=0.01,0,-0.01,-0.05$.

toward $\lambda_{0}$. Again, the shape of the curve is fairly typical. The most interesting curve is the one with the large negative initial value $x=-5 \%$. The curve starts at zero and stays virtually at zero for the first two years. This is intuitive since at the volatility 0.02 and mean reversion rate 0.1 the probability of the shadow rate moving back up above zero and the short rate becoming positive within two years is quite small. At around two-year maturity the rate of increase picks up and the curve slopes upward quite sharply between two- and fifteen-year maturity. Then the rate of increase slows and the curve gradually flattens out and tends toward $\lambda_{0} \cdot{ }^{9}$ As we shall see in Section 7 the current Japanese Government Bond yield curve has a similar shape.

\footnotetext{
${ }^{9}$ Note that the asymptotic yield is the same for all curves, as it is independent of the initial shadow rate level $x$ and is determined by the model parameters $\sigma, \theta$, and $\kappa$.
} 


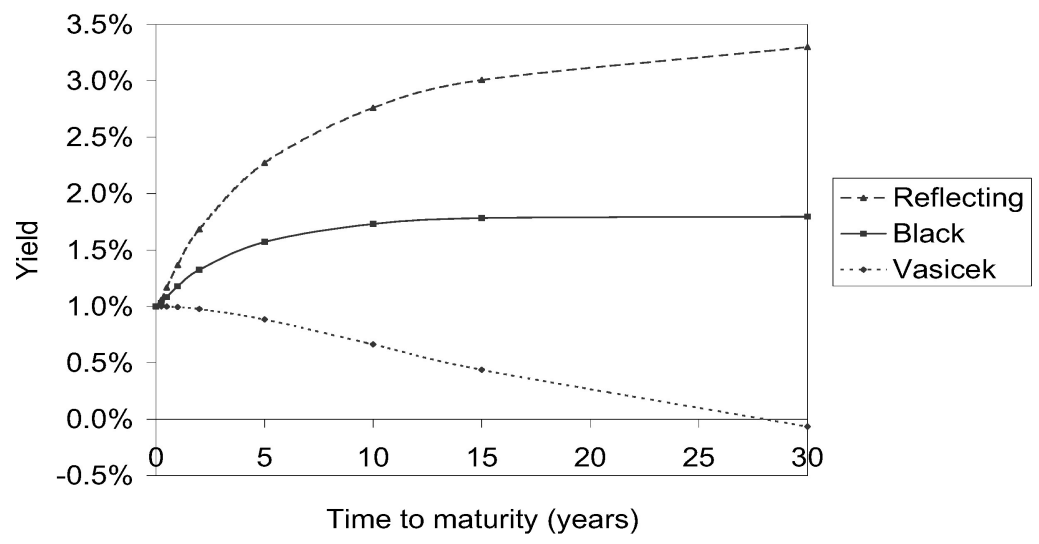

FIGURE 6.2. Yield curves in Black's model with the Vasicek shadow rate, the Vasicek process with reflecting boundary at zero, and the original Vasicek model. Parameters: $\theta=0.01, \kappa=0.1, \sigma=0.02, x=0.01$.

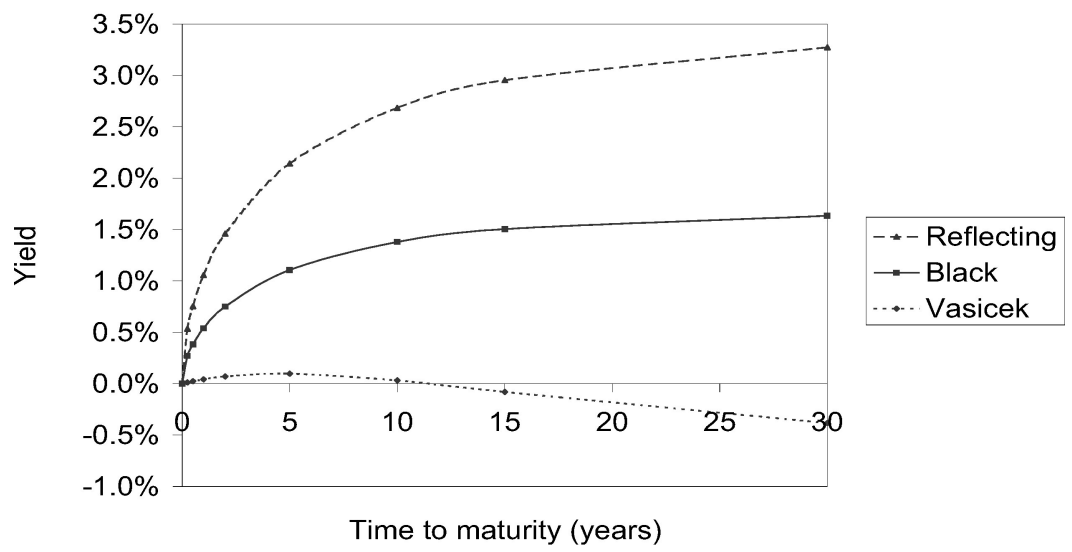

FIGURE 6.3. Yield curves in Black's model with the Vasicek shadow rate, the Vasicek process with reflecting boundary at zero, and the original Vasicek model. Parameters: $\theta=0.01, \kappa=0.1, \sigma=0.02, x=0$.

Figure 6.2 plots the yield curves in Black's model with the Vasicek shadow rate, the Vasicek process with reflecting boundary at zero, and the original Vasicek model. The initial short rate is $r=0.01$ in all three cases. The Vasicek yield curve starts at $1 \%$ and is downward sloping. It decreases toward the asymptotic yield equal to the principal eigenvalue $\lambda_{0}=\theta-\frac{\sigma^{2}}{2 \kappa^{2}}=-0.01$, which is negative for these parameter values. In contrast, the corresponding yield curves for Black's model with the Vasicek shadow rate and for the Vasicek process with reflecting boundary condition at zero are upward sloping toward their respective asymptotic yields (principal eigenvalues). The asymptotic yield for the reflecting model is significantly greater than for Black's model. Intuitively, in the reflecting model when the short rate drops to zero, it is instantaneously reflected from zero. In contrast, in Black's model the shadow rate can fall below zero. As a result, the reflecting yield curve is above Black's model curve. Figure 6.3 plots the same yield curves 
TABLE 7.1

Calibration to JGB Data

\begin{tabular}{lrrcc}
\hline Coupon & Maturity & Price & Bootstrapped yield $(\%)$ & Model yield $(\%)$ \\
\hline 4.2 & $3 / 20 / 2003$ & 104.648 & 0.02 & 0.03 \\
3.4 & $3 / 22 / 2004$ & 106.900 & 0.14 & 0.17 \\
4.4 & $3 / 21 / 2005$ & 112.729 & 0.30 & 0.36 \\
3.1 & $3 / 20 / 2006$ & 110.481 & 0.54 & 0.57 \\
2.6 & $3 / 20 / 2007$ & 109.326 & 0.76 & 0.78 \\
1.9 & $3 / 20 / 2008$ & 105.578 & 0.98 & 0.98 \\
1.9 & $3 / 20 / 2009$ & 104.723 & 1.24 & 1.16 \\
1.7 & $3 / 22 / 2010$ & 102.521 & 1.40 & 1.33 \\
1.4 & $3 / 21 / 2011$ & 99.314 & 1.51 & 1.48 \\
1.5 & $12 / 20 / 2011$ & 99.997 & 1.53 & 1.59 \\
3.8 & $9 / 20 / 2016$ & 123.287 & 2.11 & 2.09 \\
2.1 & $12 / 20 / 2021$ & 98.411 & 2.29 & 2.44 \\
2.4 & $11 / 20 / 2031$ & 94.810 & 2.88 & 2.79
\end{tabular}

Note: The first three columns give JGB data from Bloomberg, including coupon, maturity, and prices on 02/03/2002. The last two columns give boot-strapped zerocoupon yields and calibrated model yields. Calibrated Vasicek shadow rate process parameters are $\theta=0.0354, \kappa=0.212, \sigma=0.0283$, and $x=-0.0512$. JGB data source: Bloomberg.

but starting from zero. Again, the three curves tend to their respective asymptotic yields (principal eigenvalues).

\section{MODEL CALIBRATION TO THE JAPANESE GOVERNMENT BOND DATA}

In this section we calibrate Black's model with Vasicek shadow rate process to the Japanese Government Bond (JGB) data. Table 7.1 gives JGB data from Bloomber, including coupon, maturity, and prices on $02 / 03 / 2002$. The last two columns give bootstrapped zero-coupon yields and calibrated model yields. Calibrated Vasicek shadow rate process parameters are $\theta=0.0354, \kappa=0.212, \sigma=0.0283$, and $x=-0.0512$. Figure 7.1 plots JGB and calibrated model yield curves. The fit of the model to the JGB data is excellent. We have 13 data points (13 bonds), three model parameters $(\theta, \kappa$, and $\sigma)$ plus the initial shadow rate $x$. We fit the model by minimizing the root mean squared error between the JGB yield curve and the model yield curve (Table 7.1). It is particularly notable that the current implied Japanese shadow rate is negative at $-5.12 \%$.

\section{CONCLUSION}

In this paper we show that Black's model of interest rates as options has the same degree of analytical tractability as the corresponding model with reflecting boundary at zero. We develop a general framework for interest rate models of this class where the shadow rate is a one-dimensional diffusion and we derive analytical solutions for the Vasicek and shifted CIR shadow rate specifications as our principal examples. Our calculations are based on the observation that zero-coupon bonds in Black's model can be interpreted 


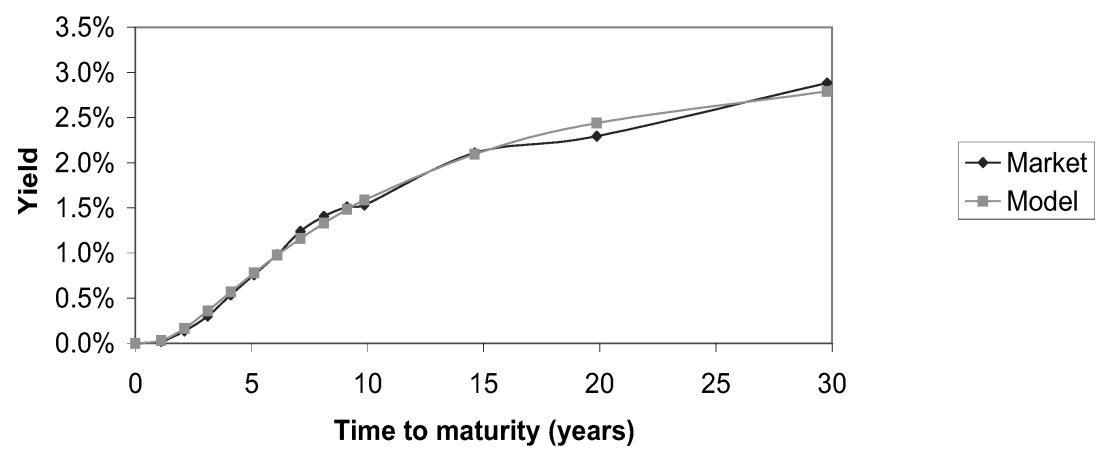

FIGURE 7.1. Japanese Government Bond yield curve on 02/03/2002 and calibrated Black's model yield curve. Calibrated Vasicek shadow rate process parameters are $\theta=0.0354, \kappa=0.212, \sigma=0.0283$, and $x=-0.0512$. JGB data source: Bloomberg.

as Laplace transforms of the area functional. We then apply spectral theory to compute them in terms of eigenfunction expansions. The same methodology is then extended to bond options and yields analytical expressions for options on zero-coupon bonds given as eigenfunction expansions. Computationally, the longer the time to maturity, the faster the eigenfunction expansions converge.

We calibrate Black's model of interest rates as options to Japanese Government Bond data and show that it is capable of providing an excellent fit to the JGB data. Not surprisingly, the current implied value of the Japanese shadow rate turns out to be negative at around $-5 \%$. The calibrated model can be used by practitioners for pricing and hedging of Japanese interest rate derivatives.

In conclusion, we note that the framework developed in this paper can be applied in other economic contexts. Generally, let $V_{t}$ be some economic variable that is not allowed to drop below a certain lower floor $L$ (e.g., a fixed price support level for a commodity). Introduce a shadow variable $X_{t}$ that is assumed to follow a process that is allowed to drop below $L$ and treat the constrained variable $V$ as an "option" on $X: V_{t}=L+\left(X_{t}-L\right)^{+}$. When the shadow variable is above the floor $L$, the constrained variable is equal to the shadow variable. When the shadow variable drops below the floor, the constrained variable stays at the floor level $L$ and will continue to stay at $L$ until the shadow variable comes back above $L$. If we are interested in the average value of the variable $V$ over some time horizon, we are led to study the distribution of an area functional $\int_{0}^{t}\left(X_{u}-L\right)^{+} d u$. These applications are left for future research.

\section{APPENDIX: PROOFS}

Proof of Proposition 3.2. The bond put payoff is $(K-P(x, t, T)) \mathbf{1}_{\{K-P(x, t, T) \geq 0\}}$. Since the zero-coupon bond price is a strictly decreasing function of the shadow rate, we can rewrite the payoff in the form $(K-P(x, t, T)) \mathbf{1}_{\left\{x \geq x^{*}\right\}}$, where $x^{*}$ is the unique root of equation (3.30). Using the eigenfunction expansion (3.24) for the zero-coupon bond, we further rewrite the payoff $\left(K-\sum_{m=0}^{\infty} c_{m} e^{-\lambda_{m}(T-t)} \varphi_{m}(x)\right) \mathbf{1}_{\left\{x \geq x^{*}\right\}}$. The expansion (3.27) now follows by specifying (3.23) to the put payoff. The coefficients of the expansion are

$$
\begin{aligned}
p_{n} & =\int_{x^{*}}^{r}\left(K-\sum_{m=0}^{\infty} c_{m} e^{-\lambda_{m}(T-t)} \varphi_{m}(x)\right) \varphi_{n}(x) \mathfrak{m}(x) d x \\
& =K \int_{x^{*}}^{r} \varphi_{n}(x) \mathfrak{m}(x) d x-\sum_{m=0}^{\infty} c_{m} e^{-\lambda_{m}(T-t)} \int_{x^{*}}^{r} \varphi_{n}(x) \varphi_{m}(x) \mathfrak{m}(x) d x .
\end{aligned}
$$


Proof of Proposition 3.3. We follow the approach of Linetsky (2002b, Sec. 5.1). Both endpoints are NONOSC and we are in the Spectral Category I. Consider the ODE (3.11) on $(l, r)$. Let $\xi_{\lambda}(x), x \in(l, r), \lambda \in \mathbb{C}$, be the unique (up to a multiple independent of $x$ ) solution such that

$$
\int_{l}^{0}\left|\xi_{\lambda}(x)\right|^{2} \mathfrak{m}(x) d x<\infty
$$

and

$$
\lim _{x \downarrow l} \frac{\xi_{\lambda}^{\prime}(x)}{\mathfrak{s}(x)}=0
$$

for each $\lambda \in \mathbb{C}$, and $\xi_{\lambda}(x)$ and $\xi_{\lambda}^{\prime}(x)$ are continuous in $x$ and $\lambda$ in $(l, r) \times \mathbb{C}$ and entire in $\lambda$ for each fixed $x \in(l, r)$. Such a solution exists by Lemma 1 in Linetsky (2002b).

Let $\eta_{\lambda}(x), x \in(l, r), \lambda \in \mathbb{C}$, be the (unique up to a multiple independent of $x$ ) solution such that

$$
\int_{0}^{r}\left|\eta_{\lambda}(x)\right|^{2} \mathfrak{m}(x) d x<\infty
$$

and

$$
\lim _{x \uparrow r} \frac{\eta_{\lambda}^{\prime}(x)}{\mathfrak{s}(x)}=0
$$

for each $\lambda \in \mathbb{C}$, and $\eta_{\lambda}(x)$ and $\eta_{\lambda}^{\prime}(x)$ are continuous in $x$ and $\lambda$ in $(l, r) \times \mathbb{C}$ and entire in $\lambda$ for each fixed $x \in(l, r)$. Such a solution exists by Lemma 1 in Linetsky (2002b).

Now consider the two intervals $(l, 0]$ and $[0, r)$ separately. Let $\psi_{\lambda}(x)$ and $\chi_{\lambda}(x)$ be two linearly independent solutions of the $\operatorname{ODE}$ (3.31) on the interval $(l, 0] ; \psi_{\lambda}(x)$ is as in Proposition 3.3 (such a solution exists by Lemma 1 in Linetsky 2002b) and $\chi_{\lambda}(x)$ is linearly independent of $\psi_{\lambda}(x)$. Let $\zeta_{\lambda}(x)$ and $\phi_{\lambda}(x)$ be two linearly independent solutions of the ODE (3.34) on the interval $[0, r) ; \phi_{\lambda}(x)$ is as in Proposition 3.3 (such a solution exists by Lemma 1 in Linetsky 2002b) and $\zeta_{\lambda}(x)$ is linearly independent of $\phi_{\lambda}(x)$ ).

Then the solutions $\xi_{\lambda}(x)$ and $\eta_{\lambda}(x)$ can be written in the form

$$
\begin{aligned}
& \xi_{\lambda}(x)=\left\{\begin{array}{ll}
\psi_{\lambda}(x), & x \in(l, 0] \\
\alpha_{1} \zeta_{\lambda}(x)+\beta_{1} \phi_{\lambda}(x), & x \in[0, r)
\end{array},\right. \\
& \eta_{\lambda}(x)=\left\{\begin{array}{ll}
\alpha_{2} \psi_{\lambda}(x)+\beta_{2} \chi_{\lambda}(x), & x \in(l, 0] \\
\phi_{\lambda}(x), & x \in[0, r)
\end{array} .\right.
\end{aligned}
$$

The continuity of $\xi_{\lambda}(x), \xi_{\lambda}^{\prime}(x), \eta_{\lambda}(x)$ and $\eta_{\lambda}^{\prime}(x)$ at $x=0$ uniquely fixes the four constants $\alpha_{1}, \alpha_{2}, \beta_{1}$, and $\beta_{2}$ :

$$
\begin{array}{ll}
\psi_{\lambda}(0)=\alpha_{1} \zeta_{\lambda}(0)+\beta_{1} \phi_{\lambda}(0), & \psi_{\lambda}^{\prime}(0)=\alpha_{1} \zeta_{\lambda}^{\prime}(0)+\beta_{1} \phi_{\lambda}^{\prime}(0), \\
\phi_{\lambda}(0)=\alpha_{2} \psi_{\lambda}(0)+\beta_{2} \chi_{\lambda}(0), & \phi_{\lambda}^{\prime}(0)=\alpha_{2} \psi_{\lambda}^{\prime}(0)+\beta_{2} \chi_{\lambda}^{\prime}(0),
\end{array}
$$

and

$$
\begin{array}{ll}
\alpha_{1}=\frac{\phi_{\lambda}(0) \psi_{\lambda}^{\prime}(0)-\psi_{\lambda}(0) \phi_{\lambda}^{\prime}(0)}{\phi_{\lambda}(0) \zeta_{\lambda}^{\prime}(0)-\zeta_{\lambda}(0) \phi_{\lambda}^{\prime}(0)}, & \beta_{1}=\frac{\psi_{\lambda}(0) \zeta_{\lambda}^{\prime}(0)-\zeta_{\lambda}(0) \psi_{\lambda}^{\prime}(0)}{\phi_{\lambda}(0) \zeta_{\lambda}^{\prime}(0)-\zeta_{\lambda}(0) \phi_{\lambda}^{\prime}(0)}, \\
\alpha_{2}=\frac{\chi_{\lambda}(0) \phi_{\lambda}^{\prime}(0)-\phi_{\lambda}(0) \chi_{\lambda}^{\prime}(0)}{\chi_{\lambda}(0) \psi_{\lambda}^{\prime}(0)-\psi_{\lambda}(0) \chi_{\lambda}^{\prime}(0)}, & \beta_{2}=\frac{\phi_{\lambda}(0) \psi_{\lambda}^{\prime}(0)-\psi_{\lambda}(0) \phi_{\lambda}^{\prime}(0)}{\chi_{\lambda}(0) \psi_{\lambda}^{\prime}(0)-\psi_{\lambda}(0) \chi_{\lambda}^{\prime}(0)}
\end{array}
$$


Then the Wronskian of $\xi_{\lambda}(x)$ and $\eta_{\lambda}(x)$ given by equations (A.5) and (A.6) is

$$
\begin{aligned}
{\left[\xi_{\lambda}, \eta_{\lambda}\right](x) \equiv } & \xi_{\lambda}(x) \frac{\eta_{\lambda}^{\prime}(x)}{\mathfrak{s}(x)}-\eta_{\lambda}(x) \frac{\xi_{\lambda}^{\prime}(x)}{\mathfrak{s}(x)} \\
& =\frac{1}{\mathfrak{s}(0)}\left(\psi_{\lambda}(0) \phi_{\lambda}^{\prime}(0)-\phi_{\lambda}(0) \psi_{\lambda}^{\prime}(0)\right)=: w(\lambda) .
\end{aligned}
$$

The Wronskian is an entire function of $\lambda$ with all of its zeros simple and located on the positive real half-line. At an eigenvalue $\lambda=\lambda_{n}$ (zero of the function $w(\lambda)$ ), the Wronskian vanishes and $\xi_{\lambda_{n}}(x)$ and $\eta_{\lambda_{n}}(x)$ become linearly dependent:

$$
\eta_{\lambda_{n}}(x)=A_{n} \xi_{\lambda_{n}}(x), \quad A_{n}=\frac{\phi_{\lambda_{n}}(0)}{\psi_{\lambda_{n}}(0)} .
$$

Thus, $\xi_{\lambda_{n}}(x)$ can be taken as a nonnormalized eigenfunction. Finally, according to Theorem 5 in Linetsky (2002b), the corresponding normalized eigenfunctions can be taken in the form

$$
\varphi_{n}(x)=\sqrt{\frac{A_{n}}{\Delta_{n}}} \xi_{\lambda_{n}}(x), \quad \Delta_{n}:=\left.\frac{d w(\lambda)}{d \lambda}\right|_{\lambda=\lambda_{n}},
$$

and we arrive at the result (3.39).

Proof of Proposition 3.4. Consider the ODE (3.34) on the interval $[0, r)$. Let $\phi_{\lambda}(x)$ be as in Proposition 3.3. Let $\zeta_{\lambda}(x)$ be the unique solution satisfying the initial conditions at zero:

$$
\zeta_{\lambda}(0)=1, \quad \zeta_{\lambda}^{\prime}(0)=0 .
$$

Select $x_{0}=0$ in the definition of the scale density (2.3) so that $\mathfrak{s}(0)=1$. The Wronskian of the two solutions is $\left[\zeta_{\lambda}, \phi_{\lambda}\right](x)=w(\lambda)$, where $w(\lambda)$ is independent of $x$. Setting $x=0$ and using the initial conditions for $\zeta$, we have $w(\lambda)=\phi_{\lambda}^{\prime}(0)$. The Wronskian is an entire function of $\lambda$ with all of its zeros simple and located on the positive real half-line. At an eigenvalue $\lambda=\lambda_{n}$ (zero of the function $\phi_{\lambda}^{\prime}(0)$ ), the Wronskian vanishes and $\phi_{\lambda_{n}}(x)$ and $\zeta_{\lambda_{n}}(x)$ become linearly dependent (recall that $\zeta_{\lambda}(0)=1$ ):

$$
\phi_{\lambda_{n}}(x)=A_{n} \zeta_{\lambda_{n}}(x), \quad A_{n}=\phi_{\lambda_{n}}(0) .
$$

Thus, $\phi_{\lambda_{n}}(x)$ can be taken as a nonnormalized eigenfunction. Finally, according to Theorem 5 in Linetsky (2002b), the corresponding normalized eigenfunction can be taken in the form

$$
\varphi_{n}(x)=\frac{\phi_{\lambda_{n}}(x)}{\sqrt{A_{n} \Delta_{n}}}, \quad \Delta_{n}:=\left.\frac{d \phi_{\lambda}^{\prime}(0)}{d \lambda}\right|_{\lambda=\lambda_{n}} .
$$

Proof of Proposition 4.1. Consider the ODE (3.31) specified to the Vasicek process:

$$
-\frac{1}{2} \sigma^{2} u^{\prime \prime}-\kappa(\theta-x) u^{\prime}=\lambda u, \quad x \in(-\infty, 0] .
$$

Introduce the standardized variable $z=\frac{\sqrt{2 \kappa}}{\sigma}(\theta-x)$ and look for solutions in the form $u(x)=e^{\frac{z^{2}}{4}} w(z)$. Substituting this functional form into (A.13), we arrive at the WeberHermite equation for $w$ (Erdelyi 1953, p. 116), and Buchholz 1969, p. 39):

$$
w_{z z}+\left(\frac{1}{2}+v-\frac{z^{2}}{4}\right) w=0, \quad v:=\frac{\lambda}{\kappa}, \quad z \in[\beta, \infty), \quad \beta:=\frac{\sqrt{2 \kappa}}{\sigma} \theta .
$$


The solution of (A.14) bounded at $z=+\infty$ is $D_{v}(z)\left(D_{v}(z)\right.$ is the Weber-Hermite parabolic cylinder function). Then the solution (4.10) of the original ODE (A.13) satisfies the square-integrability condition (3.32).

Next, consider the ODE (3.34) specified to the Vasicek process:

$$
-\frac{1}{2} \sigma^{2} u^{\prime \prime}-\kappa(\theta-x) u^{\prime}+x u=\lambda u, \quad x \in[0, \infty) .
$$

The same transformation reduces it to

$$
\begin{aligned}
& w_{z z}+\left(\frac{1}{2}+\mu-\frac{\left(\alpha-z^{2}\right)}{4}\right) w=0, \quad z \in(-\infty, \beta], \\
& \mu:=\frac{\sigma^{2}}{2 \kappa^{3}}+\frac{\lambda-\theta}{\kappa}, \quad \alpha:=\sigma \sqrt{\frac{2}{\kappa^{3}}} .
\end{aligned}
$$

The solution of (A.16) bounded at $z=-\infty$ is $D_{\mu}(\alpha-z)$, and the solution (4.11) of the original ODE (A.15) satisfies the square-integrability condition (3.35).

Finally, using the differential property of Weber-Hermite functions

$$
D_{v}^{\prime}(z)=v D_{v-1}(z)-\frac{z}{2} D_{v}(z)
$$

the function (3.37) is reduced to the form (4.12).

Proof of Proposition 5.1. Consider the ODE (3.31) for the shifted CIR process:

$$
-\frac{1}{2} \sigma^{2}(x-l) u_{x x}-\kappa(\theta-x) u_{x}=\lambda u, \quad x \in(l, 0] .
$$

Introduce the standardized variable $\xi=\frac{2 \kappa(x-l)}{\sigma^{2}}$ and look for solutions in the form $u(x)=$ $(x-l)^{-\frac{\beta}{2}} e^{\frac{k(x-1)}{\sigma^{2}}} w(\xi)$. Substituting this functional form into equation (A.18), we arrive at the Whittaker equation for $w$ (Abramowitz and Stegun 1972, p. 505; Buchholz 1969, pp. 9-20; Slater 1960, pp. 9-10):

$$
w_{\xi \xi}+\left(-\frac{1}{4}+\frac{k_{1}}{\xi}+\frac{\frac{1}{4}-m^{2}}{\xi^{2}}\right) w=0, \quad \xi \in(0, \alpha],
$$

where $k_{1}, m$, and $\alpha$ are as defined in equation (5.8). Whittaker functions $M_{k_{1}, m}(\xi)$ and $W_{k_{1}, m}(\xi)$ give two linearly independent solutions of (A.19). The functions ( $x-$ $l)^{-\frac{\beta}{2}} e^{\frac{k(x-1)}{\sigma^{2}}} M_{k_{1}, m}(\xi(x))$ and $(x-l)^{-\frac{\beta}{2}} e^{\frac{k(x-l)}{\sigma^{2}}} W_{k_{1}, m}(\xi(x))$ give two linearly independent solutions of the original ODE (A.18). To investigate the behavior of solutions near the left endpoint, we note the asymptotic behavior of Whittaker functions (Slater 1960):

$$
M_{k, m}(\xi) \sim \xi^{m+\frac{1}{2}} e^{-\frac{\xi}{2}} \text { and } W_{k, m}(\xi) \sim \xi^{-m+\frac{1}{2}} e^{-\frac{\xi}{2}} \text { as } \xi \downarrow 0 .
$$

From the asymptotics (A.20), the solution $(x-l)^{-\frac{\beta}{2}} e^{\frac{k(x-l)}{\sigma^{2}}} M_{k_{1}, m}(\xi(x))$ is both squareintegrable with the speed density (5.2) on $(l, 0]$ and satisfies the boundary condition (3.33). Thus, the solution $\psi_{\lambda}(x)$ is given by equation (5.10).

Next, consider the ODE (3.34) for the shifted CIR process:

$$
-\frac{1}{2} \sigma^{2}(x-l) u_{x x}-\kappa(\theta-x) u_{x}+x u=\lambda u, \quad x \in[0, \infty) .
$$

Introduce the standardized variable $\zeta:=\frac{\gamma \xi}{\kappa}$ and look for solutions in the form $u(x)=$ $(x-l)^{-\frac{\beta}{2}} e^{\frac{\kappa(x-l)}{\sigma^{2}}} w(\zeta)$. Substituting this functional form into (A.21), we arrive at the 
Whittaker equation for $w$ of the same form (A.19), but for the variable $\zeta$ on the interval $[\gamma \alpha / \kappa, \infty)$ and with $k_{2}$ (defined in equation (5.9)) in place of $k_{1}(m$ is the same as before). The solution square-integrable with the speed density on $[0, \infty)$ is $\phi_{\lambda}(x)=$ $(x-l)^{-\frac{\beta}{2}} e^{\frac{k(x-l)}{\sigma^{2}}} W_{k_{2}, m}(\zeta)$ (equation (5.11)).

Finally, using the differential properties of Whittaker functions (Slater 1960, p. 24, eqn. (2.4.12) and p. 25, eqn. (2.4.24)) (prime denotes differentiation in 25)

$$
\begin{gathered}
z M_{k, m}^{\prime}(z)=\left(\frac{1}{2}+m+k\right) M_{k+1, m}(z)-\left(k-\frac{z}{2}\right) M_{k, m}(z), \\
z W_{k, m}^{\prime}(z)=\left(\frac{z}{2}-k\right) W_{k, m}(z)-W_{k+1, m}(z),
\end{gathered}
$$

the function (3.37) is reduced to the form (5.12).

\section{REFERENCES}

Abramowitz, M., and I. A. Stegun (1972): Handbook of Mathematical Functions. New York: Dover.

Beaglehole, D. R. (1991): Tax Clienteles and Stochastic Processes in the Gilt Market. Ph.D. dissertation, Graduate School of Business, University of Chicago.

BlaCk, F. (1995): Interest Rates as Options, J. Finance 50, 1371-1376.

Black, F., and P. KARASINSKI (1991): Bond and Option Pricing When Short Rates Are Lognormal, Financial Anal. J., July-August, 52-59.

Borodin, A. N., and P. SAlminen (1996): Handbook of Brownian Motion. Boston. Birkhauser.

Brennan, M. J., and E. S. Schwartz (1979): A Continuous Time Approach to the Pricing of Bonds, J. Banking Finance 3, 133-155.

Buchiolz, H. (1969): The Confluent Hypergeometric Function. Berlin: Springer.

CARr, P., and R. JARrow (1990): The Stop-Loss Start-Gain Paradox and Option Valuation: A New Decomposition into Intrinsic and Time Value, Rev. Financial Stud. 3, 469-492.

CARR, P., and V. LinetSKy (2000): The Valuation of Executive Stock Options in an IntensityBased Framework, European Finance Rev. 4, 211-230.

Chesney, M., M. Jeanblanc-Picque, and M. Yor (1997): Brownian Excursions and Parisian Barrier Options, Ann. Appl. Prob. 29, 165-184.

Conley, T., L. P. Hansen, E. G. J. LutTMer, and J. Scheinkman (1997): Short-Term Interest Rates as Subordinated Diffusions, Rev. Financial Stud. 10, 525-577.

Cox, J. C., J. E. Ingersoll, and S. A. Ross (1985): A Theory of the Term Structure of Interest Rates, Econometrica 53, 385-407.

DAI, Q., and K. Singleton (2000): Specification Analysis of Affine Term Structure Models, J. Finance 55, 1943-1978.

DAvydov, D., and V. LinetSKy (2002): Structuring, Pricing and Hedging Double Barrier Step Options, J. Computat. Finance 5, Winter, 55-87.

DAvydov, D., and V. LineTSKy (2003): Pricing Options on Scalar Diffusions: An Eigenfunction Expansion Approach, Oper. Res. 51, 185-209.

DufFie, D., and R. KAN (1996): A Yield Factor Model of Interest Rates, Math. Finance 6, 379-406.

Dufresne, D. (2000): Laguerre Series for Asian and Other Options, Math. Finance 10, 407-428. 
Dufresne, D. (2001): The Integral of Geometric Brownian Motion, Adv. Appl. Prob. 33, 223 241.

Dunford, N., and J. Schwartz (1963): Linear Operators. Part II: Spectral Theory (Self-Adjoint Operators in Hilbert Spaces). New York: Wiley.

Erdelyi, A. (1953): Higher Transcendental Functions, Vol.II. New York: McGraw-Hill.

Feller, W. (1951): Two Singular Diffusion Problems, Ann. Math. 54, 173-82.

Fulton, C., S. Pruess, and Y. XIE (1996): The Automatic Classification of Sturm-Liouville Problems, Preprint (http://www.mines.edu/fs_home/spruess/papers/class.ps).

Geman, H., and M. Yor (1993): Bessel Processes, Asian Options and Perpetuities, Math. Finance $3,349-75$.

Glazman, I. (1965): Direct Methods of Qualitative Spectral Analysis of Singular Differential Operators (English translation). Jerusalem: Monson.

Goeing-Jaeschke, A., and M. Yor (2003): A Survey and Some Generalizations of Bessel Processes, Bernoulli 9, 313-349.

Goldstein, R., and W. P. Keirstead (1997): On the Term Structure of Interest Rates in the Presence of Reflecting and Absorbing Boundaries, Working paper, Ohio State University (http://fisher.osu.edu/fin/journal/dice/papers/1997/97-1.html).

Hansen, L. P., Scheinkman, J., and N. Touzi (1998): Spectral Methods for Identifying Scalar Diffusions. J. Econometrics 86, 1-32.

Heath, D., R. Jarrow, and A. Morton (1992): Bond Pricing and the Term Structure of Interest Rates: A New Methodology for Contingent Claim Valuation, Econometrica 60, 77 106.

Hull, J. (2000): Options, Futures and Other Derivatives, 4th ed. Eaglewood Cliffs, NJ: PrenticeHall.

Ito, K., and H. McKean (1974): Diffusion Processes and their Sample Paths. Berlin: Springer.

JAmes, J., and N. Weber (2000): Interest Rate Modeling. New York: Wiley.

Jamshidian, F. (1989): An Exact Bond Option Pricing Formula, J. Finance 44, 205-209.

Karatzas, I., and S. Shreve (1991): Brownian Motion and Stochastic Calculus, 2nd ed. New York: Springer.

Karlin, S., and H. M. TAYlor (1981): A Second Course in Stochastic Processes. San Diego: Academic Press.

LANGer, H., and W. S. Schenk (1990): Generalized Second-Order Differential Operators, Corresponding Gap Diffusions and Superharmonic Transformations, Math. Nachr. 148, 7-45.

Lehedev, N. N. (1972): Special Functions and Their Applications. New York: Dorer.

Levitan, B. M., and I. S. Sargsjan (1975): Introduction to Spectral Theory. Providence, RI: American Mathematical Society.

LEwIS, A. (1994): Three Expansion Regimes for Interest Rate Term Structure Models, Working paper (http://www.optioncity.net/publications.html).

Lewis, A. (1998): Applications of Eigenfunction Expansions in Continuous-Time Finance, Math. Finance 8, 349-383.

LewIS, A. (2000): Option Valuation under Stochastic Volatility. NewPort Beach Finance Press.

LiNETSKY, V. (1998): Steps to the Barrier, RISK, April, 62-65.

LinETSKY, V. (1999): Step Options, Math. Finance 9, 55-96.

Linetsky, V. (2001): Spectral Expansions for Asian (Average Price) Options, Working paper, Northwestern University (http://users.iems.nwu.edu/ linetsky/asian.pdf).

LiNETSKY, V. (2002a): Exotic Spectra, RISK, April, 85-89. 
LiNETSKy, V. (2002b): The Spectral Decomposition of the Option Value, Working paper, Northwestern University (http://users.iems.nwu.edu/ linetsky/spectral.pdf).

Linetsky, V. (2003): Computing First Hitting Time Densities for OU and CIR Processes: Applications to Mean-reverting Models, Working paper, Northwestern University (http://users.iems.nmu.edu/ linetsky/httingtime.pdf).

Lipton, A. (2001): Mathematical Methods for Foreign Exchange. Singapore: World Scientific.

McKean, H. (1956): Elementary Solutions for Certain Parabolic Partial Differential Equations, Trans. Am. Math. Soc. 82, 519-548.

Perman, M., and J. Wellner (1996): On the Distribution of Brownian Areas, Ann. Appl. Prob. 6, 1091-1111.

Pitman J., and M. Yor (1982): A Decomposition of Bessel Bridges, Z. Wahrsch. verw. Gebiete. $59,425-457$.

Rogers, L. C. G. (1995): Which Model for Term-Structure of Interest Rates Should One Use? Proceedings of IMA Workshop on Mathematical Finance, IMA Vol. 65, 93-116, New York: Springer, 93-116.

Rogers, L. C. G. (1996): Gaussian Errors, RISK 9, January, 42-45.

Shiga, T., and S. Watanabe (1973): Bessel Diffusions as a One-Parameter Family of Diffusion Processes. Z. Wahrsch. verw. Gebiete 27, 37-46.

Slater, L. J. (1960): Confluent Hypergeometric Functions. Cambridge: Cambridge University Press.

VAsiceK, O. A. (1977): An Equilibrium Characterization of the Term Structure, J. Financial Econ. 5, 177-188.

Weidmann, J. (1987): Spectral Theory of Ordinary Differential Operators, Lecture Notes in Mathematics, vol. 1258. Berlin: Springer.

Wong, E. (1964): The Construction of a Class of Stationary Markoff Processes; in Sixteenth Symposium in Applied Mathematics - Stochastic Processes in Mathematical Physics and Engineering, R. Bellman, ed. Providence, RI: American Mathematical Society, 264-276.

Yor, M. (2001): Exponential Functionals of Brownian Motion and Related Processes. Berlin: Springer. 